\title{
Geobotanical Study of the Microforests of Juniperus oxycedrus subsp. badia in the Central and Southern Iberian Peninsula
}

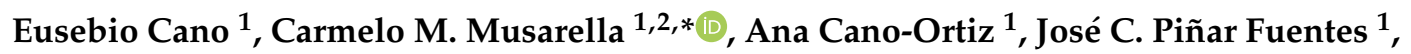 \\ Alfonso Rodríguez Torres ${ }^{3}$, Sara Del Río González ${ }^{4}$, Carlos J. Pinto Gomes ${ }^{5}$, \\ Ricardo Quinto-Canas ${ }^{6,7}$ and Giovanni Spampinato ${ }^{2}$
}

1 Department. of Animal and Plant Biology and Ecology, Section of Botany, University of Jaén, Campus Universitario Las Lagunillas s/n, 23071 Jaén, Spain; ecano@ujaen.es (E.C.); anacanor@hotmail.com (A.C.-O.); jcpfuentes@gmail.com (J.C.P.F.)

2 Department of AGRARIA, “Mediterranea” University of Reggio Calabria, Località Feo di Vito, 89122 Reggio Calabria, Italy; gspampinato@unirc.it

3 Oficina del Cambio Climático, Consejería de Agricultura, Medio Ambiente y Desarrollo Rural, Junta de Castilla-La Mancha, C/Quintanar de la Orden, s/n. 45071 Toledo, Spain; alfonsor@jccm.es

4 Department of Biodiversity and Environmental Management (Botany), Mountain Livestock Institute (CSIC-ULE), Faculty of Biological and Environmental Sciences, University of León, Campus de Vegazana s/n, 24071 León, Spain; sriog@unileon.es

5 Department of Landscape, Environment and Planning, Institute for Mediterranean Agrarian and Environmental Sciences (ICAAM), School of Science and Technology, University of Évora (Portugal), Rua Romão Ramalho, n 59, 7000-671 Évora, Portugal; cpgomes@uevora.pt

6 Faculty of Sciences and Technology, University of Algarve, Campus de Gambelas, 8005-139 Faro, Portugal; rjcanas@ualg.pt

7 Centre of Marine Sciences (CCMAR), University of Algarve, Campus de Gambelas, 8005-139 Faro, Portugal

* Correspondence: carmelo.musarella@unirc.it; Tel.: +39-0965-169-4374

Received: 15 October 2018; Accepted: 13 February 2019; Published: 20 February 2019

\begin{abstract}
We have studied Juniperus oxycedrus L. subsp. badia (H.Gay) Debeaux in the central and southern Iberian Peninsula, where the macrobioclimate ranges from Mediterranean-pluviseasonal-oceanic to Mediterranean-pluviseasonal-continental, and the thermotype from the thermo- to the supramediterranean. The relevés were taken following the Braun-Blanquet phytosociological methodology. A statistical treatment was applied to establish a separation among Juniperus communities. To understand the presence of Juniperus communities in territories dominated by species in the Quercus genus, we applied Thornthwaite's formula to calculate potential evapotranspiration. The general cluster analysis clearly distinguishes two groups of plant communities and separates the different associations in each group. All the plant communities growing on rocky crests and in extremely steep sloping areas are significantly influenced by the soil. The ombroclimatic index does not explain the presence of plant communities influenced by substrate, so we proposed a new ombroedaphoxeric index which explains the presence of Juniperus communities in territories with a thermotype between the thermo- and supramediterranean. The areas of distribution of Juniperus species are expanding due to the spread of rocky areas; this phenomenon causes an increase in edaphoxerophilous areas and a decrease in climatophilous ones. We propose four new plant associations, with updated structures and floristic compositions. Efficient conservation is possible in both the territories studied (Spain and Portugal) through the implementation of specific cross-border cooperation projects.
\end{abstract}


Keywords: Juniperion lagunae; cross-border cooperation; landscape evolution; cluster analysis; conservation; sustainable development; territorial cohesion; ombroedaphoxeric index; phytosociology; SCI areas

\section{Introduction}

There are around 60 woody species worldwide belonging to the genus Juniperus L. (Cupressaceae, gymnosperms), which is divided into three sections: J. sect. Caryocedrus Endl., J. sect. Juniperus, and J. sect. Sabina Spach [1]. The J. oxycedrus group is included within J. sect. Juniperus [2,3], and is distributed throughout the Mediterranean region, including eastern Portugal and Morocco, and extending as far as northern Iran [4]. According to Amaral Franco [4], the species J. oxycedrus L. has three clearly differentiated subspecies. J. oxycedrus L. subsp. oxycedrus occurs in the CS territories of the Iberian Peninsula, extending toward the Italian Peninsula, Sardinia, Corsica, Croatia and Slovenia [4-6]. J. oxycedrus L. subsp. macrocarpa (Sm.) Ball is widely distributed through the Mediterranean Region and W of Asia, until Syria [4,5]. Juniperus oxycedrus L. subsp. badia (H.Gay) Debeaux is restricted to Spain, Portugal and N Africa [4]. The J. oxycedrus group includes also J. navicularis Gand. (syn.: J. oxycedrus L. subsp. transtagana Franco) and J. deltoides R.P. Adams [syn.: J. oxycedrus L. subsp. deltoides (R.P. Adams) N. G. Passal], which was described and characterised as a new species by Adams and Tashev [7,8] for Greece, as distinct from J. oxycedrus. Adams and collaborators [3,9] recently reported the distribution of J. deltoides for Italy, Croatia, Greece, Turkey, Azerbaijan, Bulgaria, Cyprus, and Israel, and established phytochemical differences with J. oxycedrus due to its higher limonene and lower alpha-pinene contents. As specified previously by Adams et al. [10] and Salido et al. [11], there are clear phytochemical differences between the three subspecies of J. oxycedrus. Adams [12] also established major molecular differences between J. oxycedrus subsp. oxycedrus, J. oxycedrus subsp. badia, J. oxycedrus subsp. macrocarpa, and J. navicularis, and accordingly raises them to the rank of species. Adams et al. [13] subsequently showed that the differentiation of J. deltoides from J. oxycedrus at a level that is consistent with the divergence of J. navicularis and J. macrocarpa from J. oxycedrus is based on leaf essential oil composition, RAPD (Random Amplification of Polymorphic DNA) fingerprinting and ITS (Internal Transcribed Spacer) sequence data. Roma-Marzio et al. [14] recently proposed an identification key for the Juniperus oxycedrus group based on a combined phytochemical and morphometric approach.

Juniperus oxycedrus subsp. macrocarpa is typical of dunes and coastal sand flats and may occasionally occupy rocky areas. The communities of this taxon present on the Iberian Peninsula were described and included in the alliance Juniperion turbinatae by Rivas-Martínez [15], along with other communities dominated by Juniperus navicularis (J. oxycedrus L. subsp. transtagana Franco) and Juniperus phoenicea L. subsp. turbinata (Guss.) Nyman, also typical of psammophilous environments and dunes in coastal zones.

J. oxycedrus subsp. oxycedrus and J. oxycedrus subsp. badia are present on the Iberian Peninsula on both acid and basic hard substrates. The main differences between these two taxa according to Amaral Franco [4] mainly concern their physiognomy and the size of their mature fruits. Whereas the subspecies oxycedrus tends to take the form of a bush, the subspecies badia is a pyramid-shaped tree of considerable size. The mature galbuli in the first do not generally exceed $1 \mathrm{~cm}$ in size, while in the subspecies badia, they are over $1 \mathrm{~cm}$. Coincidentally these subspecies are frequently found coexisting in similar biotopes, which has led to frequent confusion among some authors.

Bolòs \& Vigo [16] included the var. lagunae Pau-which has the same characters as the subspecies badia- within the subspecies oxycedrus. Rivas-Martínez et al. [17], based on the work of Vicioso [18], formulated the new combination Juniperus oxycedrus L. subsp. lagunae (Pau ex C.Vicioso) Rivas Mart. [= Juniperus oxycedrus L. subsp. badia (H.Gay) Debeaux]. 
All this serves to highlight the complexity of this taxon, whose area of distribution is still insufficiently known. However, its presence in the central and southern Iberian Peninsula is very evident. In these territories it grows in formations with a broad extension, generally on rocky areas and in biotopes with shallow soils where Quercus ilex L. subsp. ballota (Desf.) Samp. (= Quercus rotundifolia Lam.) ceases to be dominant or simply cannot exist due to the lack of ecological and/or soil conditions necessary for these taxa to develop [19].

These are also phytocoenoses of considerable ecological interest owing to the presence of the companion endemics in these plant communities, which form small islands of vegetation; they act as species reservoirs as they are used for agriculture or livestock farming and have thus avoided destruction by human action. A similar condition may arise in forest fringe communities, as evidenced by Quinto-Canas et al. [20]. In these phytocoenoses it is frequent to find endemic species with varying degrees of distribution on the peninsula, such as Echinospartum ibericum, Adenocarpus argyrophyllus, Digitalis purpurea subsp. mariana, Sideritis lacaitae, Coincya longirostra, Cytisus scoparius subsp. bourgaei, Cytisus striatus subsp. eriocarpus, Genista hirsuta, G. polyanthos, Dianthus crassipes, D. lusitanus, Digitalis thapsi, D. purpurea subsp. heywoodii, D. purpurea subsp. mariana, Securinega tinctoria, Lavandula stoechas subsp. luisieri, L. stoechas subsp. sampaiana, Thymus mastichina, T. granatensis subsp. micranthus, T. zygis subsp. gracilis, and Antirrhinum graniticum subsp. onubensis [21]. These species live in sites of community interest (SCI) due to the presence of habitats such as Habitat 8220 "Siliceous rocky slopes with chasmophytic vegetation", and contain plant species including Digitali thapsi-Dianthetum lusitani Rivas-Martínez ex Fuente, Jasiono marianae-Dianthetum lusitani Rivas Goday, and Coincyo longirostraae-Dianthetum lusitani [22]. However, the dominant species in these environments is J. oxycedrus subsp. badia. These areas can therefore be classified as hotspots of interest for conservation. All these associations are included in the Habitats 2000 directive, which emphasises the ecological importance of these areas, and the need to study them for their subsequent conservation [23].

The areas dominated by Juniperus species are currently undergoing a process of expansion in response to the increase in rocky areas, which extend every year due to deforestation, forest fire, and, consequently, to soil erosion [23]. Fire is a widespread problem for the conservation of several plant communities in the Iberian Peninsula [24], leading to the spread of edaphoxerophilous zones and a decline in climatophilous ones. There are therefore more potential areas that could act as a refuge for endemic species [23].

The aim of this work was to study the communities of J. oxycedrus subsp. badia present in the central and southern Iberian Peninsula and included in Habitat 5210 "Arborescent matorral with Juniperus ssp.". This update on their structures and floristic compositions can be used to implement an efficient form of conservation for these communities.

\section{Materials and Methods}

\subsection{Study Area}

Location, Climate, Geomorphology and Soils

Juniper communities are well represented in several biogeographic units, and can be found in both the more continentalised central and eastern areas and in the more oceanic Portuguese territories, in siliceous and limestone areas. This research was therefore conducted in the central and southern Iberian Peninsula (Figure 1).

We studied 100 weather stations in the central-southern Iberian Peninsula, 29 of which have an Ombrothermic Index (IO) [25] between 3.6 and 6.3, implying that this territory has a subhumid-humid ombrotype [26]. The 71 remaining weather stations have an IO of between 2.02 and 3.6, with a predominance of a dry ombrotype throughout the whole territory. The continentality values range from 10.8 for Santiago Do Cacen (Portugal) to 21.7 in Vianos (Albacete, Spain). All this explains the presence of a Mediterranean-pluviseasonal-oceanic macrobioclimate in the westernmost areas of the territory in the study, and a Mediterranean-pluviseasonal-continental macrobioclimate in the easternmost 
territories. The thermotype ranges from thermomediterranean in the warmest territories near the Guadalquivir river valley, and supramediterranean on the crests of the Iberian plateau. However, the mean values for IO (3.89), IC (Continentality Index) [25] (18.54), and ITC (Compensated Thermicity Index) [25] (284) clearly express the territorial dominance of the dry-subhumid ombrotype, the mesomediterranean thermotype and the Mediterranean-pluviseasonal-oceanic macrobioclimate. The continental influence of the plateau is present in the easternmost areas (Jaén, Ciudad Real, and Toledo), where there is also evidence of the Mediterranean-pluviseasonal-continental macrobioclimate [23].

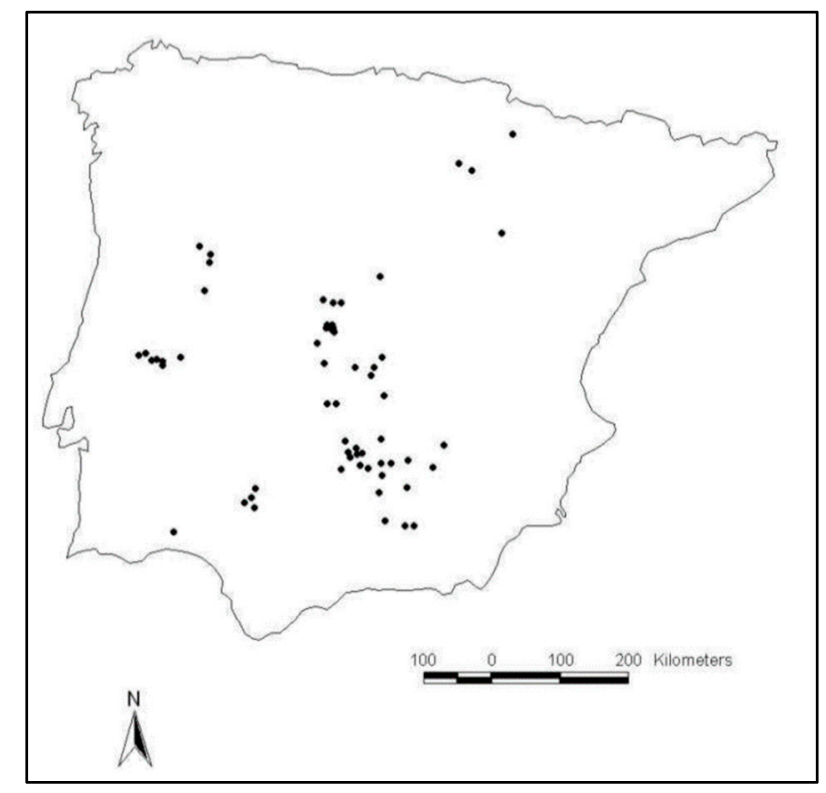

Figure 1. Study area and distribution of Juniperus oxycedrus subsp. badia on the Iberian Peninsula [19].

All these areas share the characteristic of being small mountain ranges formed by quartzite, granite, pre-Cambrian slate, limestone and dolomitic limestone, with altitudes ranging between $280-1500 \mathrm{~m}$.

\subsection{Methods}

For the nomenclature we followed References [17,18,21,27-37] for vascular plants and References [19,38] for plant communities.

We used 134 samplings taken over a wide territory (Spain and Portugal). This was done by visiting the different territories and collecting relevés from all the communities dominated by the subspecies J. oxycedrus subsp. oxycedrus and J. oxycedrus subsp. badia. Specifically, nine plant communities were studied. Among these, five have been published previously [19,39] and four are new to science.

The relevés were taken following the Braun-Blanquet phytosociological methodology, as described in works such as Braun-Blanquet [40] and Géhu \& Rivas-Martínez [41]. A relevé is a rigorous inventory of the taxa present in a study area and their degree. After compiled this inventory, the taxa coverage is evaluated by assigning a quantitative index according to the abundance-dominance and sociability scales proposed by Braun-Blanquet [40]. The abundance-dominance scale combines an estimate between the number of individuals of each existing species and the area occupied in the inventory area. The quantitative indexes (in bold) and their values are the following: +-Few individuals with very poor coverage (from $0.1 \%$ to $1 \%$ ); 1 -Very abundant individuals with low coverage (from $1 \%$ to $10 \%$ ); 2 -Individuals very abundant or covering at least $1 / 20$ of the surface (from $10 \%$ to $25 \%$ ); 3-Any number of individuals covering $\frac{1}{4}$ to $\frac{1}{2}$ of the surface (from $25 \%$ to $50 \%$ ); 4-Any number of individuals covering $\frac{1}{2}$ to $\frac{3}{4}$ of the surface (from 50\% to 75\%); 5-Any number of individuals covering more than $\frac{3}{4}$ of the surface (from $75 \%$ to $100 \%$ ). 
A statistical treatment with PAST (PAleontological STatistics) [42] and CAP ${ }^{\odot}$ (Community Analysis Package) was applied to establish a separation between Juniperus communities. We compiled an Excel $^{\odot}$ table with 134 relevés $\times 294$ species. A hierarchical clustering analysis has been applied, applying Ward's minimum variance method, using the Euclidean distance and a Detrended Correspondence Analysis (DCA). To understand the presence of Juniperus communities in territories dominated by species of the genus Quercus, we used Thornthwaite's formula, ETP monthly $=16(10 . \mathrm{T} / \mathrm{I})^{\mathrm{a}}$, to calculate potential evapotranspiration, and Montero Burgos \& González Rebollar's 0.2ETP (Potential Evapotranspiration) [43]. We prepared a new Ombroedaphoxeric Index (Ioex) with these data which justifies the presence of microforests of Juniperus species in a comparative analysis with the Ombrothermic Index (IO) proposed by Rivas-Martínez \& Loidi [25].

\section{Results}

\subsection{Phytosociological Classification Based on Numerical Analyses}

All the communities of J. oxycedrus subsp. badia share the fact that they are permanent communities with an edaphoxerophilous character, which is imposed by the rocky substrate caused by soil loss. Although the territorial ombrotype could allow the survival of Quercus species, only Q. coccifera can do so in warmer territories.

The nine communities analysed are: Juniperetum phoeniceae-badiae (JPB), Teline patentis-Pistacietum terebinthi (TP), Myrto communis-Juniperetum badiae (MJ), Echinosparto iberici-Juniperetum badiae (EJ), Cytiso eriocarpi-Juniperetum badiae (CJ), Stipo tenacissimae-Juniperetum badiae (SJ), Pistacio terebinthi-Juniperetum badiae (PJ), Genisto polyanthi-Juniperetum badiae (GJ) and Festuco merinoi-Juniperetum badiae (FJ).

The general cluster analysis clearly distinguishes two well-delimited groups: GI (FJ, MJ, SJ, EJ, GJ, CJ) (Figure 2a) and GII (JPB, TP, PJ) (Figure 2b), and separates the different associations in each group. The groups of relevés in the study belong to different plant communities, as these groups reveal clear floristic, bioclimatic, catenal and biogeographic differences, as described below.

The new DCA statistical treatments clearly separated the three associations in the subgroup: MJ, SJ and CJ. CJ was described by its authors as Cytiso eriocarpi-Juniperetum lagunae [19] for the southwest of the peninsula on siliceous substrates and in subhumid-humid environments, whereas SJ was described for the territories in the central peninsula as Stipo tenacissimae-Juniperetum lagunae [19], and shows significant floristic differences with CJ. The new association we propose in this work-MJ-is found in areas of the Sierra Morena on siliceous substrates and in dry-subhumid environments (Figure 3).

The subgroup of associations EJ and GJ grows in the Mariánico-Monchiquense sector on siliceous substrates (Paleozoic slate and quartzite), with an ombroclimate ranging from dry to humid; EJ was described by Cano et al. [19] for the supramediterranean belt as Echinosparto iberici-Juniperetum lagunae. The new association we propose is found in the mesomediterranean belt and is totally lacking in Echinospartum ibericum. The analysis of these two associations confirms their statistical separation. Both associations are in the Mariánica mountain range. The low frequency of E. ibericum explains the sole dominance of J. oxycedrus subsp. badia. The slight floristic differentiation between EJ and GJ is due to the fact that the main differentiating floristic elements, E. ibericum and Genista polyanthos, are infrequent in their respective plant communities; whereas E. ibericum is exclusive to the supramediterranean thermotype, G. polyanthos has its optimum in the thermo- and mesomediterranean, and may occasionally reach the supramediterranean, which explains the greater frequency of the microforests of Juniperus.

In contrast, the new association GJ contains species of interest such as G. polianthos, which acts as a differential species from the exclusively supramediterranean association of Echinosparto iberici-Juniperetum badiae. We therefore propose the new syntaxon Genisto polyanthi-Juniperetum badiae (Table 1 relevés from 1 to 11, typus relevé 1), located in the Marianico-Monchiquense sector. The juniper forest of J. oxycedrus subsp. badia in the eastern territories of the Iberian Peninsula (Portugal) is present in small mountain ranges with a quartzite character and frequent mesophytic flora, thanks to the 
continued prevalence of the mesomediterranean thermotype and subhumid-humid ombrotype. There is therefore a significant floristic component with an oceanic character, such as Erica arborea, Viburnum tinus and Cytisus eriocarpus, a community that has been described as Cytiso eriocarpi-Juniperetum badiae, very different from Cytiso tribracteolati-Juniperetum oxycedri which represents the edge of the cork-oak forest Teucrio baetici-Quercetum suberis, according to Pérez Latorre et al. [44].

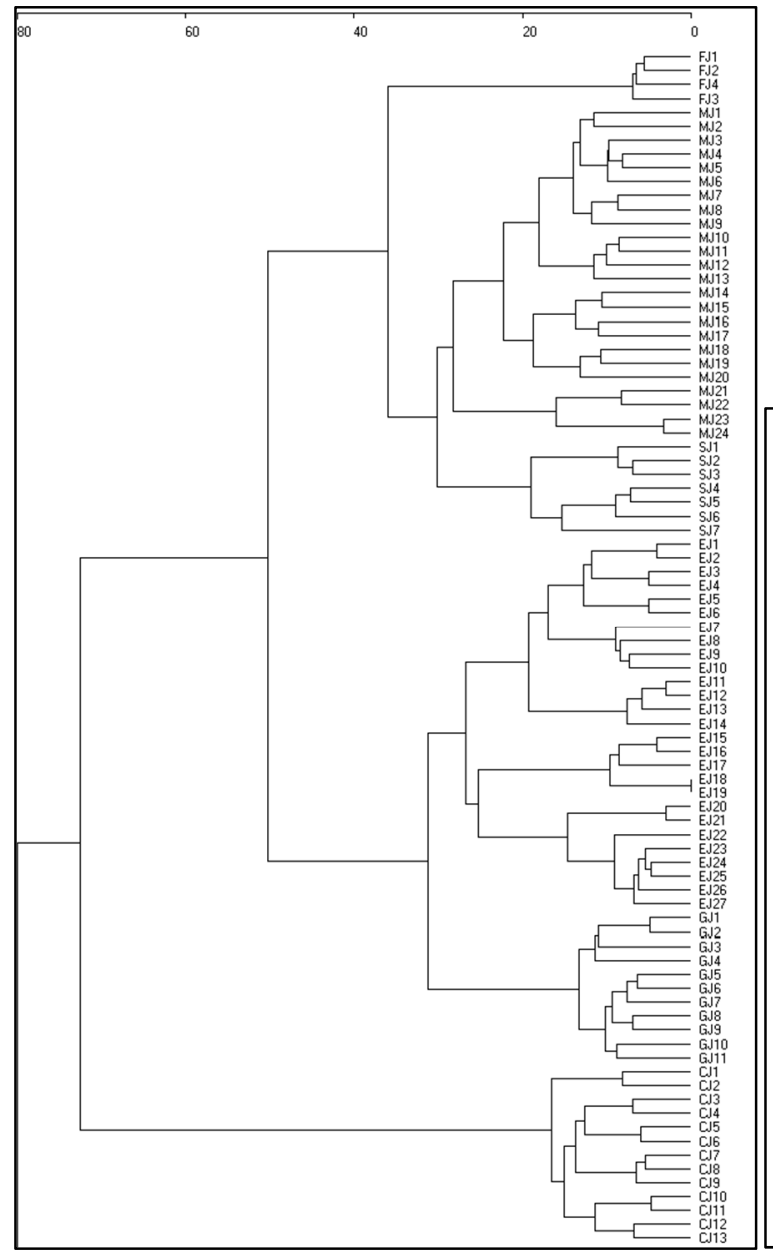

(a)

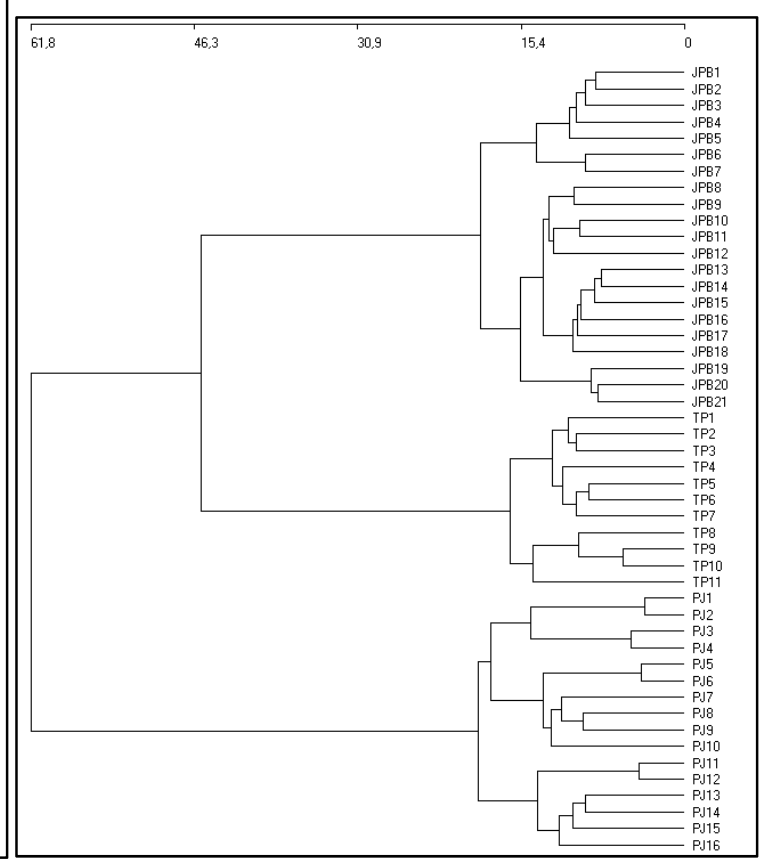

(b)

Figure 2. (a) General cluster of the six associations in group GI with Ward's method; (b) general cluster of the three associations in group GII with Ward's method. 


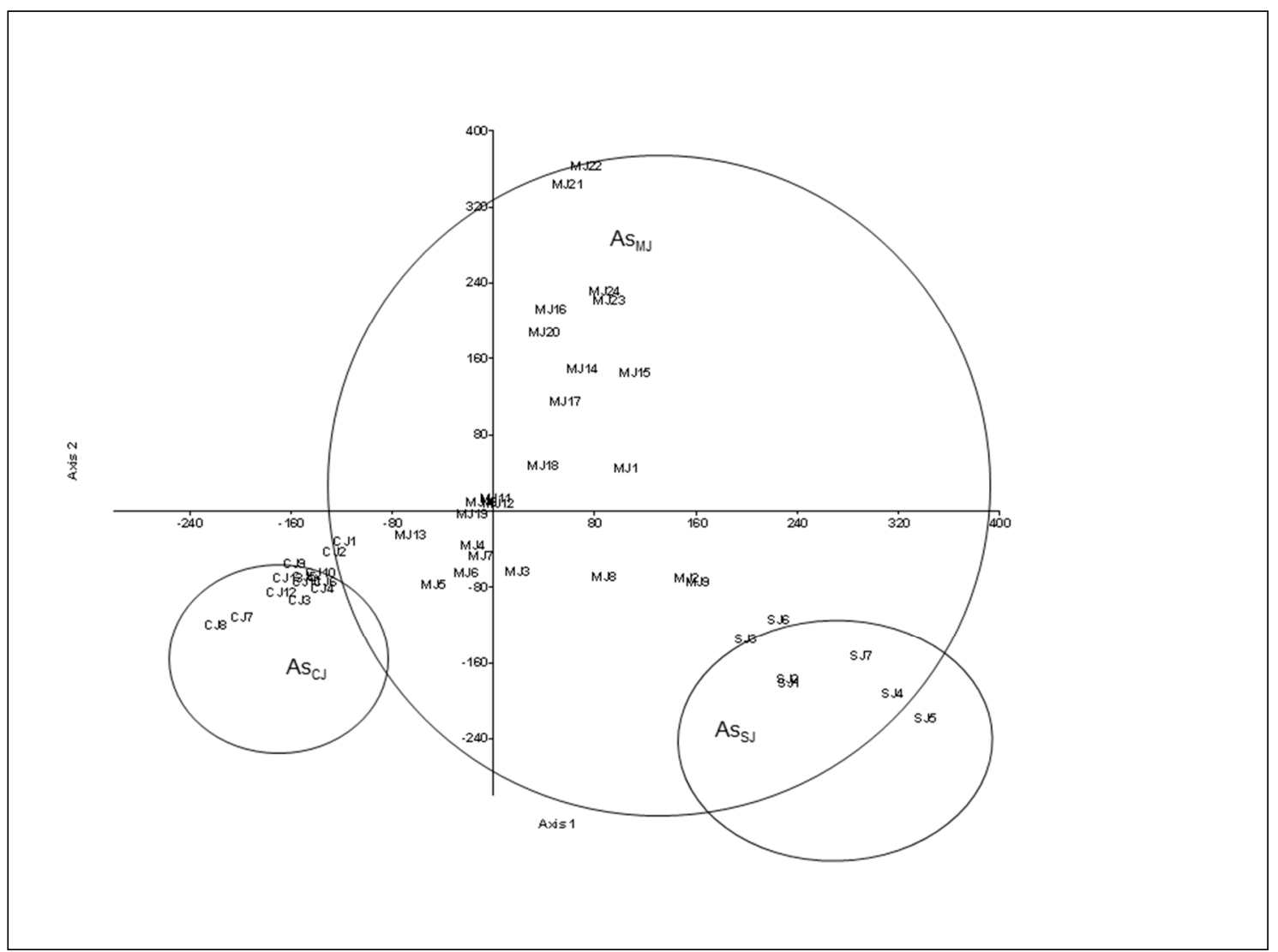

Figure 3. DCA (Detrended Correspondence Analysis) of the associations Myrto communis-Juniperetum badiae (MJ), Stipo tenacissimae-Juniperetum badiae (SJ), and Cytiso eriocarpi-Juniperetum badiae (CJ) in group GI.

Table 1. Association Genisto polyanthi-Juniperetum badiae (GJ) nova.

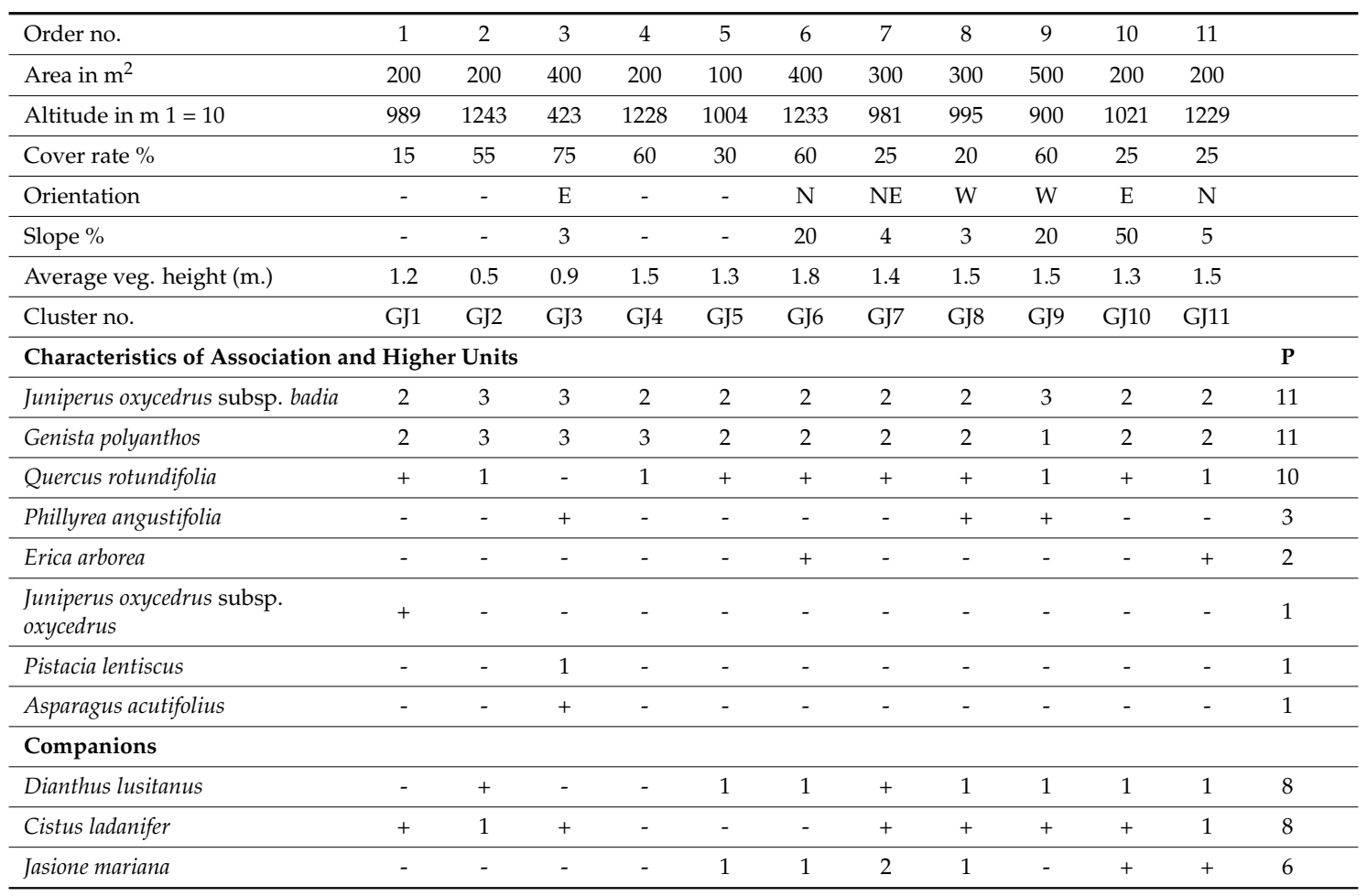


Table 1. Cont.

\begin{tabular}{|c|c|c|c|c|c|c|c|c|c|c|c|c|}
\hline Order no. & 1 & 2 & 3 & 4 & 5 & 6 & 7 & 8 & 9 & 10 & 11 & \\
\hline Arrhenatherum bulbosum & - & - & - & 1 & + & - & + & + & - & 1 & + & 6 \\
\hline Linaria saxatilis & - & - & - & + & 1 & 1 & - & + & - & 1 & - & 5 \\
\hline Sedum brevifolium & - & - & - & - & + & - & 1 & 1 & + & + & - & 5 \\
\hline Halimium ocymoides & + & + & - & - & - & + & 1 & - & - & - & - & 4 \\
\hline Rosmarinus officinalis & + & - & + & - & - & - & - & + & + & - & - & 4 \\
\hline Asphodelus albus & - & - & - & 1 & - & + & + & + & - & - & - & 4 \\
\hline Rumex angiocarpus & - & - & - & + & + & - & + & - & - & - & - & 3 \\
\hline Thymus mastichina & - & - & + & + & - & - & - & - & - & - & - & 2 \\
\hline Urginea maritima & - & - & - & - & - & - & - & + & + & - & - & 2 \\
\hline Umbilicus rupestris & - & - & - & - & + & - & - & - & - & + & - & 2 \\
\hline Dactylis lusitanica & - & - & + & + & - & - & - & - & - & - & - & 2 \\
\hline Elymus caninus & - & - & - & - & - & - & + & - & - & + & - & 2 \\
\hline Digitalis mariana & - & - & - & - & - & - & - & - & - & + & 2 & 2 \\
\hline
\end{tabular}

Other species: Cistus populifolius $x$ C. salvifolius GJ1(1), Cistus albidus GJ3(+), Retama sphaerocarpa GJ3(+), Nerium oleander GJ3(1), Flueggea tinctoria GJ3(+), Corrigiola telephiifolia GJ3(+), Scirpus holoschoenus GJ3(+), Scrophularia canina GJ3(+), Fraxinus angustifolia GJ3(+), Festuca elegans GJ4(1), Elymus hispanicus GJ4(+), Armeria capitella GJ4(1), Sedum forsteranum GJ4(+), Petrorhagia nanteuillii GJ4(+), Halimium umbellatum subsp. viscosum GJ5(+), Poa bulbosa GJ6(+), Vulpia myuros GJ7(+), Lamarckia aurea GJ7(+), Mucizonia hispida GJ8(+), Dipcadi serotinum GJ8(+), Lavandula luisieri GJ8(+), Conopodium bourgaei GJ9(+), Asplenium billotii GJ9(+), Sedum dasyphyllum GJ10(+), Geranium purpureum GJ10(+), Anogramma leptophylla GJ10(+), Stipa gigantea GJ10(+), Conopodium capillifolium GJ10(+), Narcissus rupicola GJ10(+), Festuca rothmaleri GJ10(+), Coincya longirrostra GJ11(+), Genista florida GJ11(1).

$\mathbf{P}=$ number of Presences; - = absent. Localities: GJ1. Mount Manzano. Near Torre Castañarejo (30S0449835/4256709); GJ2. Collado Grande. Torre Vigilancia (30S0443300/4257119); GJ3. Mouth of the Montoro River (30S0417604/4253109); GJ4. Pico Estrella. Finca Ruichoto (30S0448476/4250771); GJ5. Mount Manzano. Peñón de Atilano (30S0447322/4255301); GJ6. Abulagoso (30S0385725/4258301); GJ7. Cañada Real (30S0450280/4256964); GJ8. Piedrallana (30S0450483/4256122); GJ9. Mount Manzano (30S0450025/4257173); GJ10. Umbría Monroi. (30S0447629/4254779); GJ11. Collado Grande. Torre Vigilancia (30S0443228/4257178).

The community described by us as Stipo tenacissimae-Juniperetum badiae grows on carbonated and neutrobasophilous soils. Its floristic composition includes a dominance of J. oxycedrus subsp. badia and Stipa tenacissima, with other basophilous elements such as Staehelina dubia and Ruta chalepensis, together with the thermophilous elements Olea europea var. sylvestris and Osyris alba. These last are common to the new association we propose-Myrto communis-Juniperetum badiae nova-whose floristic composition comprises J. oxycedrus subsp. badia, Pistacia terebinthus, P. lentiscus, Phlomis purpurea, Myrtus communis, Aristolochia baetica, Asparagus aphyllus, and Olea europea var. sylvestris. This association is found on rocky crests on slate, quartzite and granite in thermal gorges in the Sierra Morena, and in the lower dry mesomediterranean thermotype (Table 2 rel. 1 to 24 , typus rel. 18). It is close to Stipo tenacissimae-Juniperetum badiae, but is differentiated from it by the type of substrate, thermocline, floristic composition and biogeography, as this latter association was described for eastern territories in the Toledano-Tagano sector in contact with the Manchego sector, while the association we propose here is in the Mariánico-Monchiquense sector. Group GII is formed by JPB, TP and PJ. In this case, a DCA analysis highlights the separation between the three plant communities. PJ was described for the central peninsula (Toledano-Tagano sector) on siliceous substrates, and in the dry-subhumid mesomediterranean, whereas the two new associations proposed-JPB and TP-grow on basic substrates in the Subbetic biogeographic sector, with sufficient floristic differences for the three associations to be clearly defined in the ordination analysis (Figure 4). 


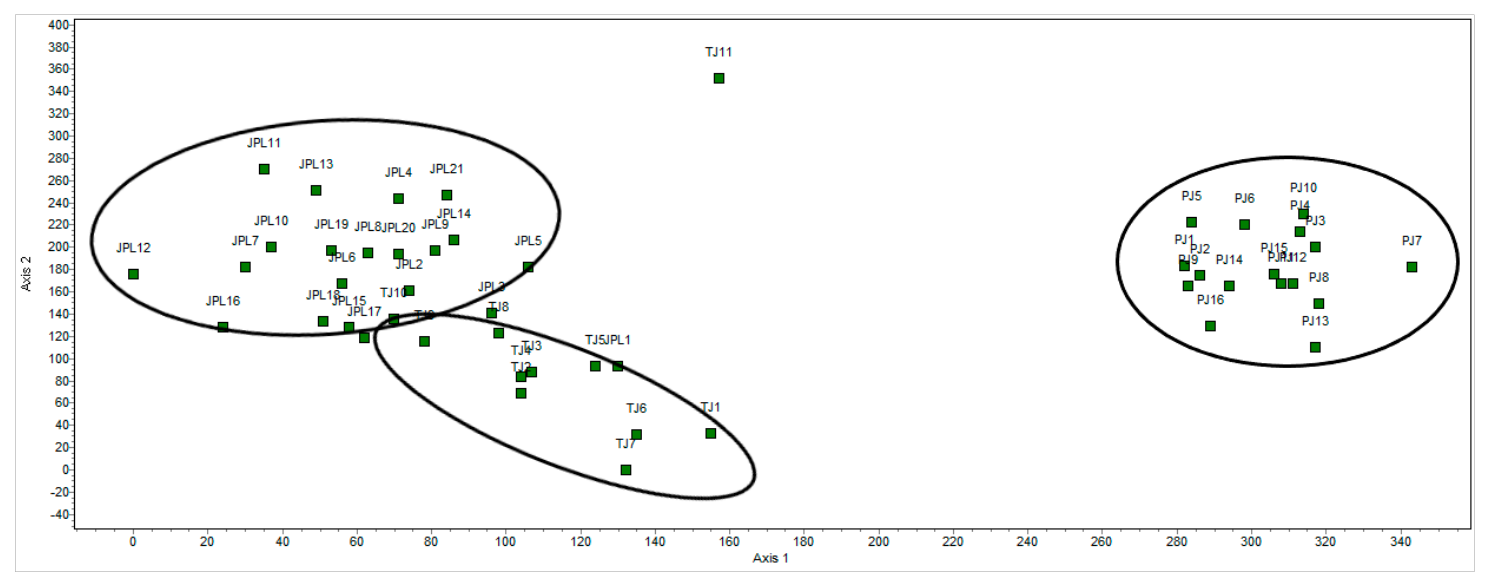

Figure 4. DCA ordination analysis of the associations Juniperetum phoeniceae-badiae (JPB), Teline patentis-Pistacietum terebinthi (TP) and Pistacio terebinthi-Juniperetum badiae (PJ) in group GII.

The subgroup $\mathrm{GII}_{\mathrm{JPB}}$, Juniperetum phoeniceae-badiae nova, represents the juniper forest with Phoenicean juniper (Juniperus phoenicea), a highly abundant plant formation in the Subbético sector, growing in subhumid mesomediterranean environments on calcareous and dolomitic limestone substrates. This is an edaphoxerophilous community with a predominance of J. oxycedrus subsp. badia, J. oxycedrus subsp. oxycedrus and J. phoenicea (Table 3 rel. 1 to 21 typus rel. 17).

Group GII includes GII ${ }_{\mathrm{PJ}}$ Pistacio terebinthi-Juniperetum badiae for Toledan territories and in the north of the province of Ciudad Real. Particularly significant are the communities of Pistacia terebinthus in the Subbético mountain ranges that grow in the subhumid-humid meso- and supramediterranean belt in rocky areas or debris fields on mountainsides. This community is physiognomically dominated by Pistacia terebintus, with other floristic elements such as J. oxycedrus subsp. badia, J. phoenicea and Teline patens. We propose the association Teline patentis-Pistacietum terebinthi nova (Table 4 relevés From 1 to 11 typus relevé 2). This association is differentiated from Phillyreo latifoliae-Pistacietum terebinthi, by Pavón Núñez et al. [45], for the absence of the thermophilous elements Clematis cirrhosa, Aristolochia baetica, Rhamnus oleoides, and R. velutinus, which are present in the typus of the association. The authors of Phillyreo latifoliae-Pistacietum terebinthi used relevés from the thermo- and mesomediterranean for their description. For this reason, relevés 3, 4 and 5 in Table 1 from the Subbético territories do not correspond to the association described. We must differentiate the thermomediterranean forest of Pistacia terebinthus from the meso- and supramediterranean forests in the Bétic biogeographic province. 
Table 2. Association Myrto communis-Juniperetum badiae (MJ) nova.

\begin{tabular}{|c|c|c|c|c|c|c|c|c|c|c|c|c|c|c|c|c|c|c|c|c|c|c|c|c|c|}
\hline Order no. & 1 & 2 & 3 & 4 & 5 & 6 & 7 & 8 & 9 & 10 & 11 & 12 & 13 & 14 & 15 & 16 & 17 & 18 & 19 & 20 & 21 & 22 & 23 & 24 & \\
\hline Area in $\mathrm{m}^{2}$ & 500 & 500 & 400 & 300 & 400 & 400 & 150 & 500 & 500 & 400 & 400 & 300 & 400 & 200 & 500 & 500 & 500 & 400 & 400 & 600 & 500 & 500 & 500 & 600 & \\
\hline Altitude in $\mathrm{m} 1=10$ & 600 & 820 & 1019 & 1006 & 568 & 988 & 240 & 820 & 780 & 912 & 832 & 766 & 842 & 1141 & 300 & 451 & 480 & 650 & 503 & 391 & 280 & 300 & 420 & 600 & \\
\hline Cover rate $\%$ & 70 & 100 & 40 & 30 & 60 & 55 & 80 & 95 & 95 & 60 & 100 & 60 & 60 & 30 & 60 & 75 & 65 & 80 & 75 & 60 & 60 & 60 & 75 & 65 & \\
\hline Slope \% & 40 & 20 & 25 & 75 & 60 & 60 & 10 & 25 & 30 & 15 & 10 & 12 & 20 & 90 & 25 & 30 & 35 & 20 & 95 & 40 & 40 & 25 & 25 & 15 & \\
\hline Average veg. height (m.) & 3.5 & 5 & 4 & 2.5 & 2.5 & 2.5 & 3 & 5 & 5 & 4 & 2.5 & 4 & 3 & 1.5 & 3.5 & 7.0 & 7.0 & 5 & 4.5 & 3:5 & 3 & 3 & 7.5 & 8.0 & \\
\hline Cluster no. & MJ1 & MJ2 & MJ3 & MJ4 & MJ5 & MJ6 & MJ7 & MJ8 & MJ9 & MJ10 & MJ11 & MJ12 & MJ13 & MJ14 & MJ15 & MJ16 & MJ17 & MJ18 & MJ19 & MJ20 & MJ21 & MJ22 & MJ23 & MJ24 & \\
\hline $\begin{array}{l}\text { Characteristics of Association and } \\
\text { Higher Units }\end{array}$ & & & & & & & & & & & & & & & & & & & & & & & & & $\mathbf{P}$ \\
\hline Juniperus oxycedrus subsp. badia & 3 & 4 & 2 & 2 & 3 & 3 & 3 & 5 & 4 & 3 & 5 & 3 & 3 & 4 & 3 & 4 & 3 & 3 & 2 & 1 & 4 & 4 & 3 & 4 & 24 \\
\hline Quercus rotundifolia & 1 & 2 & + & 1 & + & + & - & - & 1 & - & + & 1 & 1 & + & + & + & + & 1 & 1 & 1 & - & - & - & - & 17 \\
\hline Phillyrea angustifolia & 1 & 1 & - & + & 1 & 1 & 1 & 1 & - & - & + & 1 & 1 & - & - & 1 & 1 & 1 & + & + & - & - & - & - & 15 \\
\hline Pistacia terebinthus & + & + & - & - & - & - & - & - & - & - & - & - & - & 1 & 1 & 2 & 1 & - & - & + & 1 & 2 & + & + & 11 \\
\hline Myrtus communis & - & - & - & - & - & - & - & - & - & + & 1 & 3 & 2 & 1 & - & 1 & - & 2 & - & - & 1 & 1 & 1 & 1 & 11 \\
\hline Pistacia lentiscus & 1 & - & - & - & - & - & - & - & - & - & - & - & - & 1 & 1 & 1 & - & - & - & 1 & 1 & 1 & 2 & 2 & 9 \\
\hline Arbutus unedo & - & - & - & - & - & 1 & + & - & - & + & 1 & - & + & 1 & - & 1 & + & - & - & - & - & - & - & - & 8 \\
\hline Olea europea var. sylvestris & + & - & - & - & - & - & - & - & - & - & - & - & - & + & - & 2 & 2 & 3 & 4 & 3 & - & - & - & - & 7 \\
\hline Rhamnus oleoides & + & + & - & - & - & - & - & - & - & - & - & - & - & - & - & + & - & - & - & - & 2 & 2 & 2 & 2 & 7 \\
\hline Jasminum fruticans & - & - & - & - & - & - & - & - & - & - & - & - & - & - & - & + & - & - & - & + & 1 & 1 & 1 & 1 & 6 \\
\hline Erica arborea & - & - & - & - & - & - & - & - & - & + & + & + & 1 & - & - & + & - & - & - & - & - & - & - & - & 5 \\
\hline Asparagus albus & - & - & - & - & - & - & - & - & - & - & - & - & - & - & - & - & - & 2 & 1 & 2 & 1 & 1 & - & - & 5 \\
\hline Daphne gnidium & - & - & - & - & - & - & + & - & - & - & - & - & - & - & - & + & 1 & - & - & - & + & - & - & - & 4 \\
\hline Smilax aspera & - & - & - & - & - & - & - & - & - & - & - & + & - & + & - & - & - & - & - & + & - & - & + & - & 4 \\
\hline Phlomis purpurea & - & - & - & - & - & - & - & - & - & - & - & - & - & - & + & + & - & - & - & - & 1 & 2 & - & - & 4 \\
\hline Osyris alba & - & + & - & - & - & + & - & - & + & - & - & - & - & - & - & - & - & - & - & - & - & - & - & - & 3 \\
\hline Juniperus oxycedrus subsp. oxycedrus & + & - & - & - & 2 & 1 & - & - & - & - & - & - & - & - & - & - & - & - & - & - & - & - & - & - & 3 \\
\hline Rhamnus alaternus & - & - & - & - & - & - & - & - & - & - & - & - & - & - & - & 1 & 1 & - & - & - & 1 & - & - & - & 3 \\
\hline Quercus broteroi & - & - & - & - & - & - & - & - & - & - & - & - & - & - & + & + & + & - & - & - & - & - & - & - & 3 \\
\hline Asparagus acutifolius & - & 1 & - & - & - & - & - & - & - & - & - & - & - & - & - & - & + & - & - & - & - & - & - & - & 2 \\
\hline Thapsia villosa & - & - & - & - & - & - & + & - & - & - & - & - & - & - & - & - & - & + & - & - & - & - & - & - & 2 \\
\hline Phillyrea latifolia & - & - & - & - & - & - & - & - & - & - & - & - & - & - & - & - & - & - & - & + & 1 & - & - & - & 2 \\
\hline
\end{tabular}


Table 2. Cont.

\begin{tabular}{|c|c|c|c|c|c|c|c|c|c|c|c|c|c|c|c|c|c|c|c|c|c|c|c|c|c|}
\hline Order no. & 1 & 2 & 3 & 4 & 5 & 6 & 7 & 8 & 9 & 10 & 11 & 12 & 13 & 14 & 15 & 16 & 17 & 18 & 19 & 20 & 21 & 22 & 23 & 24 & \\
\hline Asparagus aphyllus & - & - & - & - & - & - & - & - & - & - & - & - & - & - & - & - & - & - & - & - & 1 & 1 & - & - & 2 \\
\hline Rubia peregrina & - & - & - & - & - & - & - & - & - & - & - & - & - & - & - & - & 1 & - & - & - & - & - & - & - & 1 \\
\hline Viburnum tinus & - & - & - & - & - & - & - & - & - & - & + & - & - & - & - & - & - & - & - & - & - & - & - & - & 1 \\
\hline Pyrus bourgaeana & - & - & - & - & - & - & - & - & - & - & - & - & - & - & - & - & + & - & - & - & - & - & - & - & 1 \\
\hline Teucrium fruticans & - & - & - & - & - & - & - & - & - & - & - & - & - & - & - & - & - & - & - & - & - & + & - & - & 1 \\
\hline Pistacia $x$ saportae & - & - & - & - & - & - & - & - & - & - & - & - & - & - & - & - & - & - & - & + & - & - & - & - & 1 \\
\hline Quercus suber & - & - & - & - & + & - & - & - & - & - & - & - & - & - & - & - & - & - & - & - & - & - & - & - & 1 \\
\hline Quercus marianica & - & - & - & - & - & - & - & - & - & - & + & - & - & - & - & - & - & - & - & - & - & - & - & - & 1 \\
\hline Quercus canariensis & - & - & - & - & - & - & - & - & - & - & - & + & - & - & - & - & - & - & - & - & - & - & - & - & 1 \\
\hline \multicolumn{26}{|l|}{ Companions } \\
\hline Cistus ladanifer & - & - & - & + & + & + & 2 & - & 2 & 1 & + & 1 & 1 & 1 & 1 & - & 1 & + & + & + & 1 & - & - & - & 16 \\
\hline Rosmarinus officinalis & - & - & - & - & - & - & - & + & 2 & 1 & + & 2 & + & - & 1 & - & + & + & + & + & + & + & - & - & 13 \\
\hline Cistus albidus & + & - & - & - & - & + & - & - & - & - & - & - & - & 1 & 1 & + & 1 & - & - & + & + & + & + & + & 11 \\
\hline Cheilanthes maderensis & - & + & 1 & + & 1 & - & - & - & - & + & - & - & 1 & - & - & - & - & + & 1 & - & - & - & + & + & 10 \\
\hline Urginea maritima & - & + & - & - & - & - & - & - & 1 & + & + & + & + & + & - & - & - & + & - & + & - & - & - & - & 9 \\
\hline Lavandula sampaiana & - & - & - & - & + & + & + & + & + & - & - & - & - & - & - & 1 & - & - & 1 & - & - & + & - & - & 8 \\
\hline Umbilicus rupestris & - & + & 1 & - & + & + & - & - & - & + & - & - & + & - & - & - & - & + & + & - & - & - & - & - & 8 \\
\hline Sedum brevifolium & - & - & 1 & - & - & + & - & - & - & + & - & - & + & - & - & - & - & + & - & - & - & - & - & - & 5 \\
\hline Mucizonia hispida & - & - & - & - & - & - & - & - & - & + & - & - & + & - & + & - & - & + & + & - & - & - & - & - & 5 \\
\hline Asphodelus albus & - & - & - & - & - & + & 1 & + & + & + & - & - & - & - & - & - & - & - & - & - & - & - & - & - & 5 \\
\hline Bryonia cretica & - & - & - & - & - & - & - & - & - & - & - & - & - & - & - & + & + & + & - & - & - & - & + & + & 5 \\
\hline Astragalus lusitanicus & - & - & - & - & - & - & + & - & - & + & + & 1 & + & - & - & - & - & - & - & - & - & - & - & - & 5 \\
\hline Dactylis hispanica & - & + & - & - & - & - & - & - & + & - & - & - & - & - & - & - & - & - & - & - & - & - & + & + & 4 \\
\hline Jasione mariana & - & - & - & + & - & + & - & - & - & - & - & - & 1 & - & - & - & - & - & - & + & - & - & - & - & 4 \\
\hline Asplenium ceterach & - & + & 1 & - & - & - & - & - & - & - & - & - & - & - & + & - & - & + & - & - & - & - & - & - & 4 \\
\hline Arrhenatherum bulbosum & - & - & - & - & - & + & - & - & + & - & - & + & + & - & - & - & - & - & - & - & - & - & - & - & 4 \\
\hline Halimium atriplicifolium & - & - & - & - & - & - & - & - & - & - & - & - & - & 1 & + & + & + & - & - & - & - & - & - & - & 4 \\
\hline Digitalis heywoodii var. albicans & - & - & - & - & - & - & - & - & - & - & - & - & - & 1 & 1 & + & + & - & - & - & - & - & - & - & 4 \\
\hline Sedum dasyphyllum & + & - & 1 & 1 & - & - & - & - & - & - & - & - & - & - & - & - & - & - & - & - & - & - & - & - & 3 \\
\hline
\end{tabular}


Table 2. Cont.

\begin{tabular}{|c|c|c|c|c|c|c|c|c|c|c|c|c|c|c|c|c|c|c|c|c|c|c|c|c|c|}
\hline Order no. & 1 & 2 & 3 & 4 & 5 & 6 & 7 & 8 & 9 & 10 & 11 & 12 & 13 & 14 & 15 & 16 & 17 & 18 & 19 & 20 & 21 & 22 & 23 & 24 & \\
\hline Thymus mastichina & - & - & - & - & - & - & - & - & + & - & - & - & - & - & 1 & - & - & - & - & - & + & - & - & - & 3 \\
\hline Sanguisorba minor & - & - & - & - & - & - & - & - & - & - & + & - & - & - & - & - & - & - & - & - & - & - & + & + & 3 \\
\hline Tamus communis & - & - & + & - & - & - & - & - & - & - & + & - & - & - & - & - & 1 & - & - & - & - & - & - & - & 3 \\
\hline Nerium oleander & - & - & - & - & - & - & - & - & - & - & - & - & - & + & - & + & - & - & - & 1 & - & - & - & - & 3 \\
\hline Anogramma leptophylla & - & - & - & + & - & - & - & - & - & - & - & - & - & - & + & - & - & + & - & - & - & - & - & - & 3 \\
\hline Selaginella denticulata & - & - & - & - & - & - & - & - & - & - & - & - & - & - & + & - & - & - & - & - & - & - & + & + & 3 \\
\hline Cytisus scoparius subsp. bourgaei & - & - & - & - & - & - & - & - & - & - & - & - & - & - & - & 1 & - & - & - & - & 1 & 2 & - & - & 3 \\
\hline Halimium umbellatum subsp. viscosum & - & - & 1 & - & - & 2 & - & - & - & - & - & - & - & - & - & - & - & - & 1 & - & - & - & - & - & 3 \\
\hline Genista hirsuta & - & 1 & - & - & - & - & - & - & - & - & - & + & - & - & - & - & - & + & - & - & - & - & - & - & 3 \\
\hline Lavandula luisieri & - & - & - & - & - & - & - & + & - & - & - & + & + & - & - & - & - & - & - & - & - & - & - & - & 3 \\
\hline Cistus salvifolius & - & 1 & - & - & - & - & - & - & - & - & + & + & - & - & - & - & - & - & - & - & - & - & - & - & 3 \\
\hline
\end{tabular}

Other species: Coincya longirrostra MJ7, MJ15(+), Sedum sediforme MJ1, MJ9(+), Retama sphaerocarpa MJ9, MJ20(+), Vitis vinifera subsp. sylvestris MJ14, MJ16(+), Adenocarpus argyrophyllus MJ4, MJ5(+), Erica scoparia MJ7(2), MJ8(+), Halimium ocymoides MJ7(1), MJ13(+), Cytisus striatus subsp. eriocarpus MJ2(1), MJ19(2), Adenocarpus telonensis MJ2(1), MJ8(+), Rubus ulmifolius MJ1(+), MJ20(+), Digitalis mariana MJ20, Flueggea tinctoria MJ21(+), Dianthus crassipes MJ21(1), Linaria saxatilis MJ6(+), Calluna vulgaris MJ13(1), Cheilanthes hispanica MJ4(+), Helichrysum serotinum MJ9(+), Teucrium gnaphalodes MJ2(+), Teucrium pseudochamaepitys MJ9(+), Cheilanthes tinaei MJ2(+), Stipa capensis MJ10(+), Hyacinthoides hispanica MJ3(+), Pterocepalus diandrus MJ13(+).

$\mathbf{P}=$ number of Presences; - = absent. Localities: MJ1. Mora de Toledo (Toledo) [19], (Stipo tenacissimae-Juniperetum lagunae); MJ2. Mora de Toledo (Toledo) [19], (Stipo tenacissimae-Juniperetum lagunae); MJ3. Collado Sierra de la Solana (30S0404372/4259526); MJ4. Puerto Viejo (30S0382806/4254598); MJ5. Crestones cuerda sierra Chillón (30S0334946/4289958); MJ6. Sierra de Solana (30S0404372/4259526); MJ7. Aldea Cerezo al Yeguas (Cardeña); MJ8. Puerto Lapice (C.Real) [19], (Stipo tenacissimae-Juniperetum lagunae); MJ9. Marjaliza (Toledo) [19], (Stipo tenacissimae-Juniperetum lagunae); MJ10. Sierrra Madrona (30S04067709/4250513); MJ11. Sierra Quintana (oeste) (30S0391176/4250840); MJ12. Swamp of the Garganta (30S0374625/4260280); MJ13. Camino Peña Escrita-Finca Valmayor (30S0387688/4255453); MJ14 Puerto Viejo (30S0382806/4254598); MJ15. Aldea Cerezo al Yeguas (Cardeña); MJ16. Aldea Cerezo al Yeguas (Cardeña); MJ17. Aldea Cerezo al Yeguas (Cardeña); MJ18. Near San Benito

(30S0352613/4271155); MJ19. Easternmost Sierra Almadén (30S0346378/4291566); MJ20. Mouth of the Montoro River (30S0417854/4253846); MJ21. Garganta del Río Viar (Sevilla) [19], (Community of Phlomis purpurea); MJ22. Garganta del Río Viar (Sevilla) [19], (Community of Phlomis purpurea); MJ23. Garganta del Río Viar (Sevilla) [19], (Community of Phlomis purpurea); MJ24. Garganta del Río Viar (Sevilla) [19], (Community of Phlomis purpurea). 
Table 3. Association Juniperetum phoeniceo-badiae (JPB) nova.

\begin{tabular}{|c|c|c|c|c|c|c|c|c|c|c|c|c|c|c|c|c|c|c|c|c|c|c|}
\hline Order no. & 1 & 2 & 3 & 4 & 5 & 6 & 7 & 8 & 9 & 10 & 11 & 12 & 13 & 14 & 15 & 16 & 17 & 18 & 19 & 20 & 21 & \\
\hline Area in $\mathrm{m}^{2}$ & 150 & 250 & 150 & 250 & 250 & 250 & 200 & 400 & 150 & 200 & 400 & 400 & 500 & 150 & 500 & 250 & 250 & 200 & 600 & 500 & 600 & \\
\hline Altitude in $\mathrm{m} 1=10$ & 50 & 105 & 95 & 69 & 105 & 95 & 115 & 135 & 140 & 115 & 115 & 120 & 105 & 110 & 105 & 113 & 120 & 157 & 130 & 100 & 95 & \\
\hline Cover rate $\%$ & 40 & 70 & 80 & 65 & 60 & 60 & 40 & 65 & 50 & 75 & 65 & 65 & 70 & 45 & 65 & 70 & 75 & 60 & 60 & 60 & 60 & \\
\hline Orientation & $\mathrm{N}$ & $\mathrm{W}$ & SW & $S$ & $\mathrm{~N}$ & $\mathrm{~S}$ & $\mathrm{~N}$ & E & NW & $\mathrm{E}$ & SE & SE & $\mathrm{S}$ & SW & $\mathrm{S}$ & SW & SW & $S$ & $S$ & SW & SW & \\
\hline Slope $\%$ & 40 & 15 & 25 & 15 & 15 & 25 & 25 & 20 & 15 & 15 & 14 & 10 & 20 & 25 & 20 & 15 & 15 & 20 & 45 & 20 & 15 & \\
\hline Average veg. height (m.) & 2.0 & 2.5 & 2.5 & 2.0 & 2.0 & 2.5 & 3.0 & 2.5 & 1.8 & 4.0 & 2.5 & 4.0 & 2.5 & 2.0 & 2.5 & 2.5 & 2.0 & 2.0 & 4.0 & 2.0 & 1.8 & \\
\hline Cluster no. & JPB1 & JPB2 & JPB3 & JPB4 & JPB5 & JPB6 & JPB7 & JPB8 & JPB9 & JPB10 & JPB11 & JPB12 & JPB13 & JPB14 & JPB15 & JPB16 & JPB17 & JPB18 & JPB19 & JPB20 & JPB21 & \\
\hline $\begin{array}{l}\text { Characteristics of Association and } \\
\text { Higher Units }\end{array}$ & & & & & & & & & & & & & & & & & & & & & & $\mathbf{P}$ \\
\hline Juniperus phoenicea & 1 & - & - & 1 & 1 & 2 & 2 & + & 3 & 4 & 3 & 3 & 3 & 1 & 2 & 3 & 3 & 3 & 2 & 3 & 1 & 19 \\
\hline Juniperus oxycedrus subsp. badia & + & 3 & 4 & 3 & 3 & 3 & 3 & 3 & 1 & 1 & + & 2 & 3 & 2 & 3 & 3 & 3 & 2 & 3 & 3 & 3 & 21 \\
\hline $\begin{array}{l}\text { Juniperus oxycedrus subsp. } \\
\text { oxycedrus }\end{array}$ & - & 1 & 1 & - & - & - & - & 2 & 2 & 1 & 1 & 1 & 2 & 2 & 3 & 2 & 2 & + & - & 1 & 3 & 15 \\
\hline Quercus rotundifolia & - & + & - & - & + & 1 & + & + & + & + & 1 & + & - & - & - & 1 & + & + & + & + & + & 15 \\
\hline Pistacia terebinthus & - & - & + & - & - & - & - & - & - & + & - & + & - & + & - & - & - & - & + & - & - & 5 \\
\hline Pistacia lentiscus & 1 & - & - & 1 & - & - & - & - & - & - & - & - & 1 & + & - & - & - & - & - & - & - & 4 \\
\hline Quercus coccifera & 1 & - & - & + & 1 & 1 & - & - & - & - & + & - & 1 & + & - & - & - & - & - & - & - & 7 \\
\hline Asparagus acutifolius & + & - & - & + & - & - & - & - & - & - & + & - & - & - & - & - & - & - & - & - & - & 3 \\
\hline Jasminum fruticans & + & - & - & - & - & + & - & - & - & - & - & - & - & 1 & 1 & - & - & - & - & - & - & 4 \\
\hline Daphne gnidium & - & + & - & 1 & + & - & 1 & - & - & - & 1 & - & + & - & + & + & - & - & 1 & + & + & 11 \\
\hline Quercus faginea & - & + & - & 1 & - & - & + & - & - & - & + & - & - & - & - & - & - & - & + & - & - & 5 \\
\hline Thapsia villosa & - & + & - & - & + & - & + & + & + & - & + & + & - & - & - & + & - & + & - & - & - & 9 \\
\hline Carex hallerana & - & - & 1 & - & - & - & - & - & - & - & - & - & + & - & + & - & - & - & - & 1 & - & 4 \\
\hline Rhamnus myrtifolius & - & - & 1 & - & - & - & - & 1 & - & - & - & + & - & - & - & - & - & 2 & - & - & - & 4 \\
\hline Pinus halepensis & - & - & - & + & - & - & - & - & - & - & - & + & - & + & - & - & - & - & - & - & - & 3 \\
\hline Phillyrea latifolia & - & - & - & - & - & - & - & - & - & - & - & - & - & - & + & - & - & - & - & - & - & 1 \\
\hline Pinus salzmannii & - & - & - & - & - & - & - & - & - & - & - & - & - & - & - & - & - & + & - & + & - & 2 \\
\hline $\begin{array}{l}\text { Lonicera periclymenum subsp. } \\
\text { hispanica }\end{array}$ & - & - & - & - & - & - & - & - & - & - & - & - & - & - & - & - & - & + & - & - & - & 1 \\
\hline Ptilostemon hispanicus & - & - & - & - & - & - & - & + & - & - & - & - & - & - & - & - & - & - & + & - & - & 2 \\
\hline Hedera ibernica & - & - & - & - & - & - & - & - & - & - & - & - & - & - & - & - & + & + & - & - & - & 2 \\
\hline
\end{tabular}


Table 3. Cont

\begin{tabular}{|c|c|c|c|c|c|c|c|c|c|c|c|c|c|c|c|c|c|c|c|c|c|c|}
\hline Order no. & 1 & 2 & 3 & 4 & 5 & 6 & 7 & 8 & 9 & 10 & 11 & 12 & 13 & 14 & 15 & 16 & 17 & 18 & 19 & 20 & 21 & \\
\hline Rubia peregrina & - & - & - & - & - & - & - & - & - & - & - & - & - & - & - & - & - & - & - & + & + & 2 \\
\hline Bupleurum rigidum & - & - & - & - & - & - & - & - & - & - & - & - & - & - & - & - & - & - & + & - & - & 1 \\
\hline Ilex aquifolium & - & - & - & - & - & - & - & - & - & - & - & - & - & - & - & - & - & - & + & - & - & 1 \\
\hline Clematis flammula & - & - & - & - & - & - & - & - & - & - & - & - & - & - & - & - & - & - & + & - & - & 1 \\
\hline Rhamnus alaternus & + & - & - & - & - & - & - & - & - & - & - & - & - & - & - & - & - & - & - & - & - & 1 \\
\hline Smilax aspera & - & - & - & + & - & - & - & - & - & - & - & - & - & - & - & - & - & - & - & - & - & 1 \\
\hline Teline patens & - & - & - & - & 2 & - & - & - & - & - & - & - & - & - & - & - & - & - & - & - & - & 1 \\
\hline Melica minuta & - & - & - & - & - & - & - & - & - & - & - & - & - & - & - & - & - & - & + & - & - & 1 \\
\hline Paeonia broteroi & - & - & - & - & + & - & - & - & - & - & - & - & - & - & - & - & - & - & - & - & - & 1 \\
\hline \multicolumn{23}{|l|}{ Companions } \\
\hline Rosmarinus officinalis & + & 2 & 2 & - & 1 & 1 & - & - & - & - & - & - & + & + & - & 1 & 1 & - & 1 & + & - & 11 \\
\hline Thymus orospedanus & - & 1 & 1 & - & - & + & 2 & 2 & - & - & - & + & - & - & - & + & + & - & + & - & + & 10 \\
\hline Asphodelus albus & - & + & + & - & - & - & 1 & + & + & + & 1 & + & - & - & - & + & - & - & - & - & - & 9 \\
\hline Phlomis lychnitis & - & - & - & - & - & - & + & 1 & + & + & + & 1 & - & + & + & - & - & - & - & - & + & 9 \\
\hline Brachypodium retusum & - & - & - & - & + & + & - & + & - & + & - & 1 & 1 & - & + & - & - & - & - & + & + & 9 \\
\hline Thymus mastichina & - & - & - & + & - & - & 1 & - & + & + & 2 & 1 & + & - & - & - & 1 & - & - & - & + & 9 \\
\hline Cistus albidus & - & - & - & - & - & 1 & - & 2 & + & - & - & + & + & + & - & 1 & - & - & - & - & - & 7 \\
\hline Dactylis hispanica & - & - & - & - & + & + & - & + & + & - & - & - & + & + & - & - & - & - & - & - & - & 6 \\
\hline Lavandula latifolia & - & 1 & + & - & 1 & + & - & - & - & - & - & - & - & - & - & - & - & - & + & - & - & 5 \\
\hline Thymus zygis subsp. gracilis & - & 1 & - & - & - & - & - & - & - & - & - & - & 1 & 1 & 1 & - & - & - & - & + & - & 5 \\
\hline Cistus monspeliensis & - & + & - & 2 & - & + & - & - & - & - & - & - & + & + & - & - & - & - & - & - & - & 5 \\
\hline Teucrium capitatum & - & + & - & - & - & - & - & + & 1 & - & - & - & - & - & - & - & + & - & - & - & - & 4 \\
\hline Crataegus laciniata & + & - & - & 1 & 1 & - & - & - & - & - & - & - & - & - & - & - & + & - & - & - & - & 4 \\
\hline Aphyllantes monspeliensis & - & - & - & + & - & - & - & - & + & - & 1 & - & - & - & - & - & - & - & + & - & - & 4 \\
\hline Urginea maritima & - & - & - & - & - & - & - & + & - & - & - & + & - & + & + & - & - & - & - & - & - & 4 \\
\hline Halimium atriplicifolium & - & 1 & - & + & + & - & - & - & - & - & - & - & - & - & - & - & - & - & - & - & - & 3 \\
\hline Eryngium campestre & - & - & - & - & - & - & + & + & + & - & - & - & - & - & - & - & - & - & - & - & - & 3 \\
\hline Teucrium rotundifolium & - & - & + & - & - & - & - & - & - & - & + & - & - & - & - & - & - & - & - & - & - & 2 \\
\hline
\end{tabular}


Table 3. Cont.

\begin{tabular}{|c|c|c|c|c|c|c|c|c|c|c|c|c|c|c|c|c|c|c|c|c|c|c|}
\hline Order no. & 1 & 2 & 3 & 4 & 5 & 6 & 7 & 8 & 9 & 10 & 11 & 12 & 13 & 14 & 15 & 16 & 17 & 18 & 19 & 20 & 21 & \\
\hline Rosa canina & - & - & - & - & + & - & - & - & - & - & - & - & - & - & - & - & + & - & - & - & - & 2 \\
\hline Berberis hispanica & - & - & - & - & - & - & - & + & - & - & - & - & - & - & - & - & - & 1 & - & - & - & 2 \\
\hline Retama sphaerocarpa & - & - & - & + & + & - & - & - & - & - & - & - & - & - & - & - & - & - & - & - & - & 2 \\
\hline Helianthemum syriacum & - & - & - & - & - & - & 2 & + & - & - & - & - & - & - & - & - & - & - & - & - & - & 2 \\
\hline $\begin{array}{l}\text { Helianthemum cinereum subsp. } \\
\text { rotundifolium }\end{array}$ & - & - & - & - & - & - & - & - & + & + & - & - & - & - & - & - & - & - & - & - & - & 2 \\
\hline Erinacea anthyllis & - & - & - & - & - & - & - & - & 2 & - & - & - & - & - & - & - & - & - & - & + & - & 2 \\
\hline Helictotrichon filifolium & - & - & - & - & - & - & - & - & - & - & + & - & - & - & - & - & - & + & - & - & - & 2 \\
\hline Genista scorpius & - & - & - & - & - & - & - & - & - & - & - & - & 1 & - & - & - & - & - & - & - & 1 & 2 \\
\hline Crocus serotinus subsp. salzmannii & - & - & - & - & - & - & - & - & - & - & - & - & + & - & + & - & - & - & - & - & - & 2 \\
\hline Genista boissieri & - & - & - & - & - & - & - & - & - & - & - & - & - & - & - & + & - & - & - & + & - & 2 \\
\hline Santolina canescens & - & - & - & - & - & - & - & - & - & - & - & - & - & - & - & 1 & - & - & - & - & + & 2 \\
\hline
\end{tabular}

Other species: Rubus ulmifolius JPB1(+), Phagnalon saxatile JPB1(+), Linum tenue JPB3(1), Helianthemum ledifolium JPB3(+), Buxus sempervirens JPB9(2), Filipendula vulgaris JPB7(+), Plantago lanceolata JPB7(+), Ornithogalum narbonense JPB7(+), Polygala monspeliaca JPB7(+), Ophrys tenthrediniferfa JPB7(+), Anthyllis vulneraria subsp. maura JPB2(+), Arrhenatherum album JPB8(+), Fumana paradoxa JPB9(1), Lotus

corniculatus JPB9(1), Sanguisorba minor JPB9(+), Crupina crupinastrum JPB10(+), Helleborus foetidus JPB10(+), Geranium purpureum JPB11(+), Narcissus assoanus JPB11(+), Scorpiurus muricatus JPB12(+), Sherardia corniculatus JPB9(1), Sanguisorba minor JPB9(+), Crupina crupinastrum JPB10(+), Helleborus foetidus JPB10(+), Geranium purpureum JPB11(+), Narcissus assoanus JPB11(+), Scorpiurus muricatus JPB12(+), Sherardia
arvensis JPB12(+), Medicago rigidula JPB12(+), Crepis vesicaria subsp. haenseleri JPB12(+), Bellis perennis JPB12(+), Echinaria capitata JPB12(+), Poa annua JPB12(+), Rumex bucephalophorus JPB12(+), Verbuscum

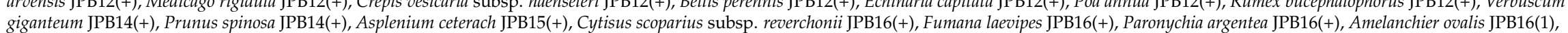

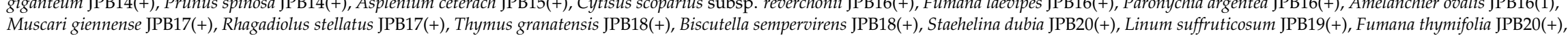
Muscari giennense JPB17(+), Rhagadiolus stellatus JPB17(+)
Lonicera splendida JPB20(+), Leuzea conifera JPB20(+).

P = number of Presences; - = absent. Localities: JPB1.—Casilla de los Rajones. JPB2 and JPB5.—Alto de las Muelas. JPB3.—Los Yegüerizos. JPB4.—Bujaraiza. JPB6.-Los Palancares. JPB7._La Fresnedilla. JPB8.—Collado de la Traviesa. JPB9 and JPB18.—Lancha de la Escalera. JPB10.—La Morra. JPB11._Fuente del Milano. JPB12.—Hoya de Miguel Barba. JPB13 and JPB17._Vilches. JPB14.—La Canaleja. JPB15.-Aguascebas Reservoir. JPB16.-San Antón. JPB19.-Rise to the birth of the Guadalquivir. JPB20 and JPB21._-Highway Quesada-Pozo Alcón. 
Table 4. Association Teline patentis-Pistacietum terebinthi (TP) nova.

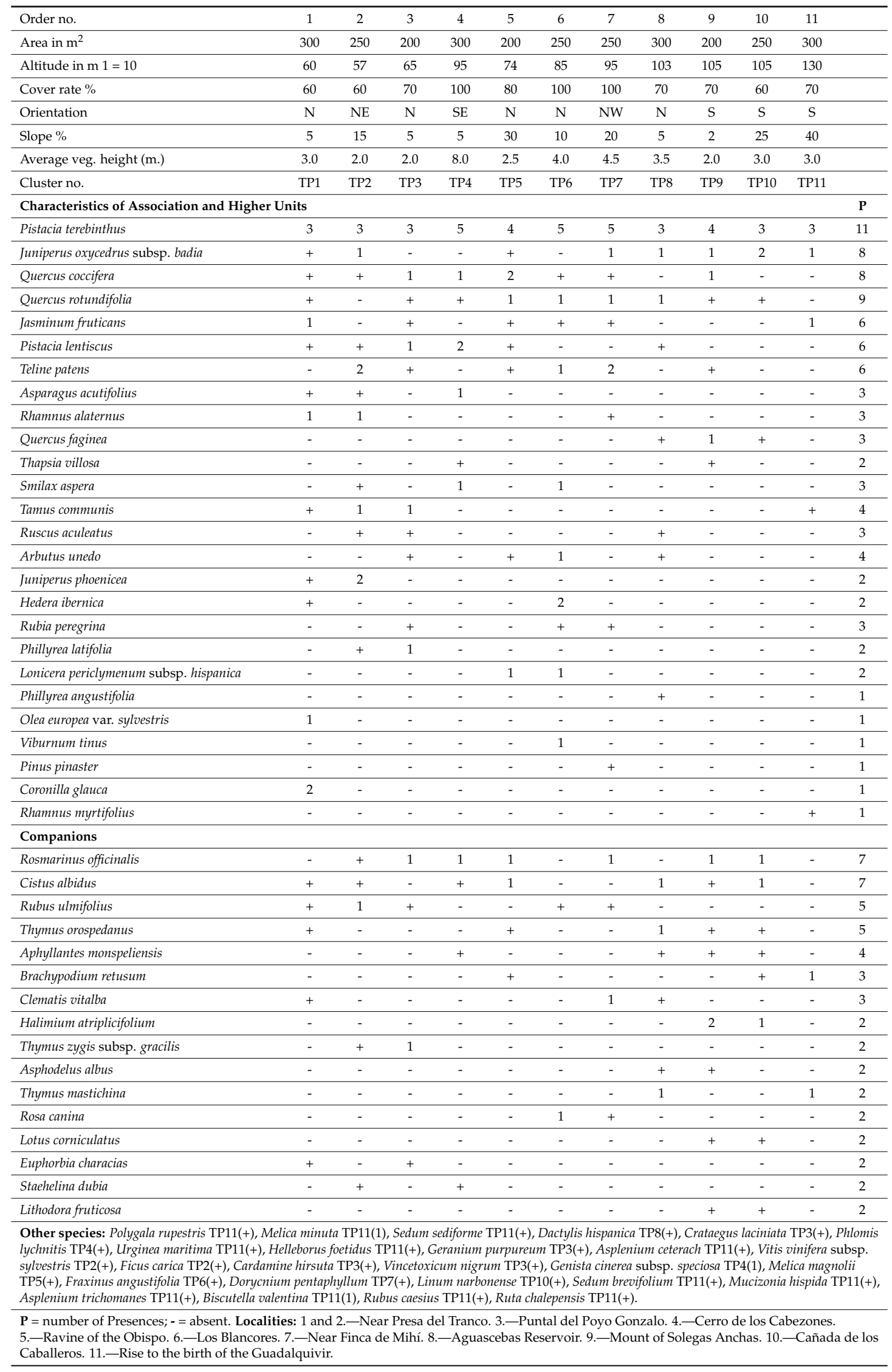




\subsection{Synthetic Vegetation Analysis}

The synthetic table (Appendix A) reveals a differential floristic composition between all the associations studied, with a predominance of species of Pistacio lentisci-Rhamnetalia alaterni Rivas-Martínez such as J. oxycedrus subsp. oxycedrus, J. oxycedrus subsp. badia, Pistacia lentiscus, P. terebinthus, Quercus coccifera, Phillyrea latifolia, P. angustifolia, Rhamnus alaternus, R. lycioides, R. oleoides, Pinus halepensis, Arbutus unedo and Asparagus albus. The floristic composition of the associations allows us to include them in the alliance Juniperion badiae, a thermo- to supramediterranean dry juniper scrub found in the Luso-Extremaduran province in the central Iberian Peninsula $[19,38]$.

\subsection{Catenal Analysis of the Landscape Evolution}

Territories behave differently in response to the general climate, the type of substrate and the topography of the terrain. For this reason, areas on rocky crests-even though they may be in rainy environments and surrounded by climactic forests-behave differently from the territories around them. In these circumstances, islands evolve which may contain edaphoseries, minoriseries, and permaseries [46-48]. All the plant communities growing on rocky crests and in steeply sloping areas are very significantly influenced by the soil, which allows their existence. All territories have a substrate and an orography which determines whether they have a greater or lesser capacity to retain water. There are special substrates such as ultramafic rocks (serpentines) that are rich in heavy cations and have a high content of ferromagnesium minerals [49]. The Betic mountains (southern Spain) comprise marble limestone, gypsum, and serpentines [50]. The rainfall values for the serpentine territories (Sierra Bermeja) indicate a wet ombrotype, very like the precipitations for the mountains in northwestern Serbia [51]. The xericity of serpentines often gives rise to forests and scrublands that do not correspond to the ombrotype in the territory: plants living here develop ecophysiological and morpho-anatomical adaptations to withstand the limitations [52]. In ideal situations with good soil texture and structure and without slopes, we can assume that the water retention (WR) is maximum (100\%). Otherwise there are losses due to run-off and drainage, and the WR may therefore vary. Water is also lost through potential evapotranspiration (ETP). However, as plants have the capacity to self-regulate their losses, it can be assumed that the residual evapotranspiration e $=0.2 \mathrm{ETP}$. So two parameters (i.e., e and WR) are implicated in the development of a vegetation that is essentially conditioned by rainfall. The Ombroclimatic Index (IO) does not therefore explain the presence of plant communities that are influenced by the substrate, and we propose the new Ombroedaphoxeric Index (Ioex) to explain the presence of communities of Juniperus in territories with a thermo- to supramediterranean thermotype.

$$
\text { Ioex }=\mathrm{P}_{\mathrm{p}}-\mathrm{e} / \mathrm{T}_{\mathrm{p}} * \mathrm{WR},
$$

$\mathrm{P}_{\mathrm{p}}=$ Annual positive precipitation; $\mathrm{T}_{\mathrm{p}}=$ Annual positive temperature [25]; $\mathrm{e}=$ residual evapotranspiration whose value is $0.2 \mathrm{ETP}$ [43]; $\mathrm{WR}=$ water retention in parts per unit, whose values may be $0.25,0.50,0.75$, and 1 .

Table 5 shows the values for PP, TP, and IO according to the criterion established by Reference [52]. The value of ETP is obtained by applying Thornthwaite's formula, $\mathrm{ETP}_{\text {monthly }}=16(10 . \mathrm{T} / \mathrm{I})^{\mathrm{a}}$, where "T" is the mean monthly temperature, " $\mathrm{I}$ " is the annual heat index, and "a" is a parameter that depends on the values taken by "I". 
Table 5. Comparative value of indices IO and Ioex in some localities in the Southern Iberian Peninsula.

\begin{tabular}{|c|c|c|c|c|c|c|c|c|c|c|}
\hline Weather Station & PP & TP & IO & Ombrotype & ETP & e & Ioex1 & Ioex $2^{*}$ & Ioex3 & $\begin{array}{c}\text { Ombroclimatic Behaviour } \\
\text { of the Locality }\end{array}$ \\
\hline Almadén-Minas (CR) & 625.2 & 194.4 & 3.21 & dry & 808.54 & 161.7 & 0.59 & 1.19 & 1.78 & Semiarid \\
\hline Cabezas Rubias (H) & 993.4 & 177.6 & 5.59 & subhumid & 702.14 & 140.42 & 1.2 & 2.4 & 3.6 & dry \\
\hline Aracena $(\mathrm{H})$ & 1025.8 & 175.2 & 5.85 & subhumid & 703.46 & 140.69 & 1.26 & 2.52 & 3.78 & dry \\
\hline Santiago Pontones (J) & 1148.7 & 164.4 & 6.98 & subhumid & 675.23 & 135.04 & 1.54 & 3.08 & 4.62 & dry \\
\hline Vadillo Castril (J) & 1182.2 & 140.4 & 8.42 & humid & 488.88 & 97.72 & 1.93 & 3.86 & 5.79 & subhumid \\
\hline Grazalema $(\mathrm{Ca})$ & 1962.2 & 183.6 & 10.7 & humid & 726.22 & 145.24 & 2.47 & 4.94 & 7.42 & subhumid \\
\hline Montoro (Co) & 522.4 & 210 & 2.48 & dry & 903.15 & 180.63 & 0.4 & 0.81 & 1.22 & arid \\
\hline Pozoblanco (Co) & 514.4 & 193.2 & 2.66 & dry & 805.45 & 161.09 & 0.45 & 0.91 & 1.37 & arid \\
\hline $\begin{array}{l}\text { Villanueva del } \\
\text { Arzobispo (J) }\end{array}$ & 698.2 & 196.8 & 3.54 & dry & 915.7 & 183.14 & 0.65 & 1.3 & 1.96 & semiarid \\
\hline
\end{tabular}

$\mathrm{PP}=$ Positive precipitation of the year [25]; TP = Positive temperature of the year [25]; e = residual evapotranspiration whose value is 0.2 ETP [43]; ETP = potential evapotranspiration; Ioex1, Ioex2, Ioex3= values of Ioex when WR is $0.25,0.50$, and $0.75 ;{ }^{*}=$ most representative value of Ioex.

If we apply the formula Ioex for the assumptions that WR is $0.25,0.50$ and 0.75 , we obtain three values, of which the most representative is Ioex 2 . Table 5 establishes the equivalence values in such a way that although the territorial bioclimate allows the existence of climactic forests, in wild areas with $\mathrm{WR}=50 \%$ the humid ombrotype becomes dry or subhumid depending on whether the value of $\mathrm{WR}=25 \%$ or $50 \%$. The subhumid becomes dry and the dry becomes semiarid or arid. Therefore, areas with IO $>8$ have Ioex 2 values of 3.86 and 4.94, which is equivalent to subhumid. This allows the presence of an edaphoxerophilous community of Quercus faginea s.l. or Abies pinsapo in rocky areas, as occurs in Grazalema (Cadiz), and a value of Ioex $1=2.47$ in the case that WR $=25 \%$. There is an edaphoxerophilous community of Quercus ilex subsp. ballota in this situation in Cazorla (Jaén) and in Grazalema (Cadiz). When the underlying ombrotype is subhumid, the equivalence value of Ioex2 is dry; an underlying dry IO gives semiarid and even arid values of Ioex 2 if the underlying horizon is less than dry. This does not allow the development of Quercus tree species, but does allow the genus Juniperus. The value of Ioex is affected by climate change, as evidenced by Del Río et al. [53]. According to these authors, this change in annual rainfall redistribution is taking place heterogeneously, and decreasing in most of the mountainous areas of Grazalema, Ronda, Cazorla, Segura, Sierra Nevada and a large part of the Sierra Morena. However, they have detected an increase in rainfall on the Andalusian coast and particularly in Almería. This affects forest stands, and-together with human activity [23]-favours a redistribution of the current forests due to a decline in the forests of Quercus and an increase in the microforests of Juniperus.

\section{Discussion}

J. oxycedrus L. subsp. badia (H. Gay) Debeaux and J. oxycedrus L. subsp. lagunae (Pau ex Vicioso) Rivas-Martínez have been used indistinctly as a result of accumulated and persistent errors [19,38,54]. According to Cano-Ortiz et al. [53], the name J. oxycedrus L. subsp. lagunae (Pau ex Vicioso) Rivas-Martínez is invalid, and its correct name is J. oxycedrus L. subsp. badia (H. Gay) Debeaux. For this reason, we rectify here the association names of: Echinosparto iberici-Juniperetum lagunae with Echinosparto iberici-Juniperetum badiae, Cytiso eriocarpi-Juniperetum lagunae with Cytiso eriocarpi-Juniperetum badiae and Stipo tenacissimae-Juniperetum lagunae with Stipo tenacissimae-Juniperetum badiae (see the syntaxonomical scheme below).

The following figures show the catenal contacts and reveal the coexistence of plant communities with different ombroclimatic demands. In the catenas in Figures 5 and 6 (Sierra Morena) with opposing orientations and on a siliceous substrate, there is an ombroclimatic gradient from dry to humid from the base of the mountain to the summit. In this case the presence of microforests of Juniperus is only possible due to the influence of the substrate; this is repeated in the catenas in Figures 7 and 8 (Cazorla and Mágina), which have calcareous substrates and a northern orientation, implying higher rainfall than in Sierra Morena. We therefore find edaphoxerophilous copses of Quercus ilex subsp. ballota and 
juniper ("sabinares"), and holm oak forests ("enebrales") of Juniperetum phoeniceae-badiae, along with forests of Portuguese oak ("quejigares") and "acerales" of Viburno tini-Quercetum alpestris, Berberido hispanicae-Quercetum alpestris and Daphno latifoliae-Aceretum granatensis.

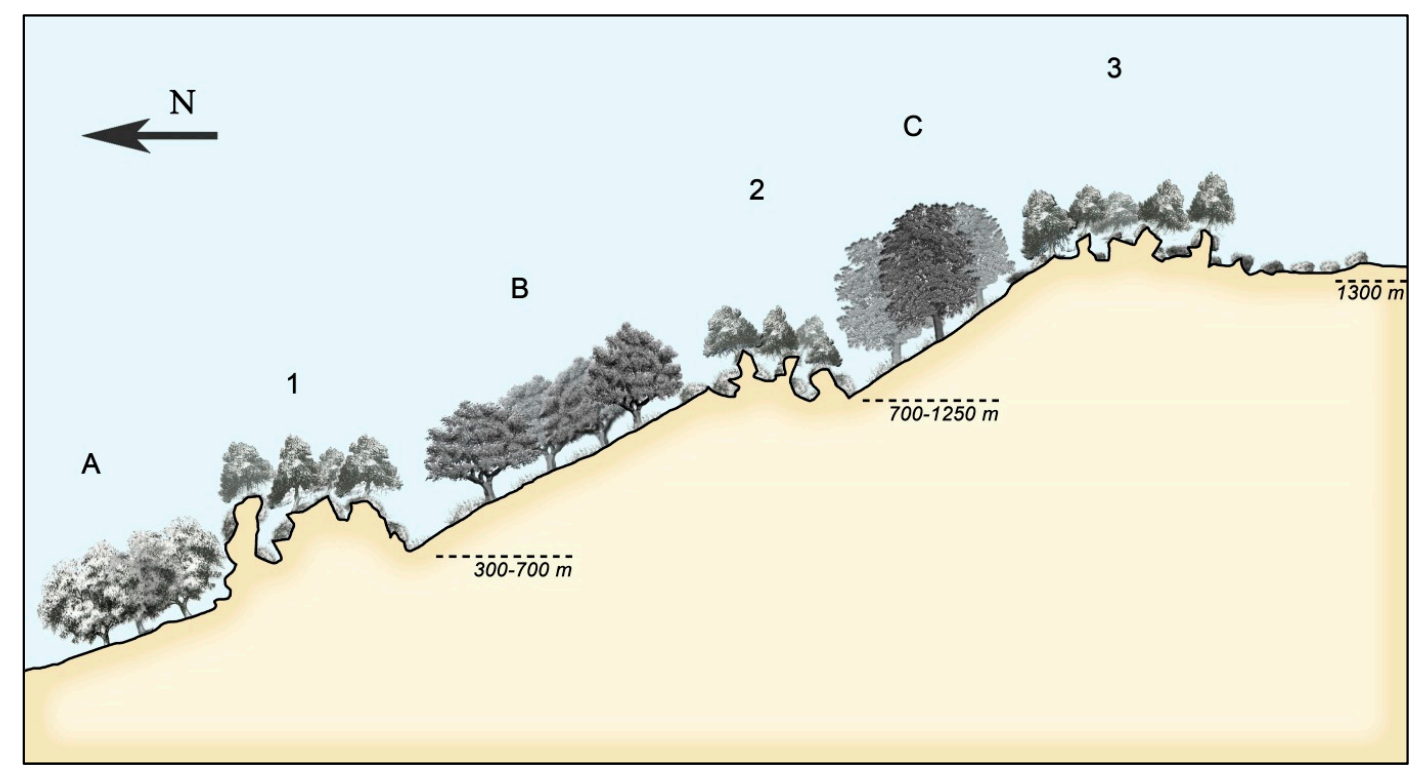

Figure 5. A, B, and C: climatic forests of A) Myrto communis-Quercetum rotundifoliae; B) Poterio agrimonioidis-Quercetum suberis; C) Arbuto unedonis-Quercetum pyrenaicae. 1, 2, and 3: edaphoxerophilous microforests of Juniperus sp. 1) Myrto communis-Juniperetum badiae; 2) Genisto polianthi-Juniperetum badiae; 3) Echinosparto iberici-Juniperetum badiae.

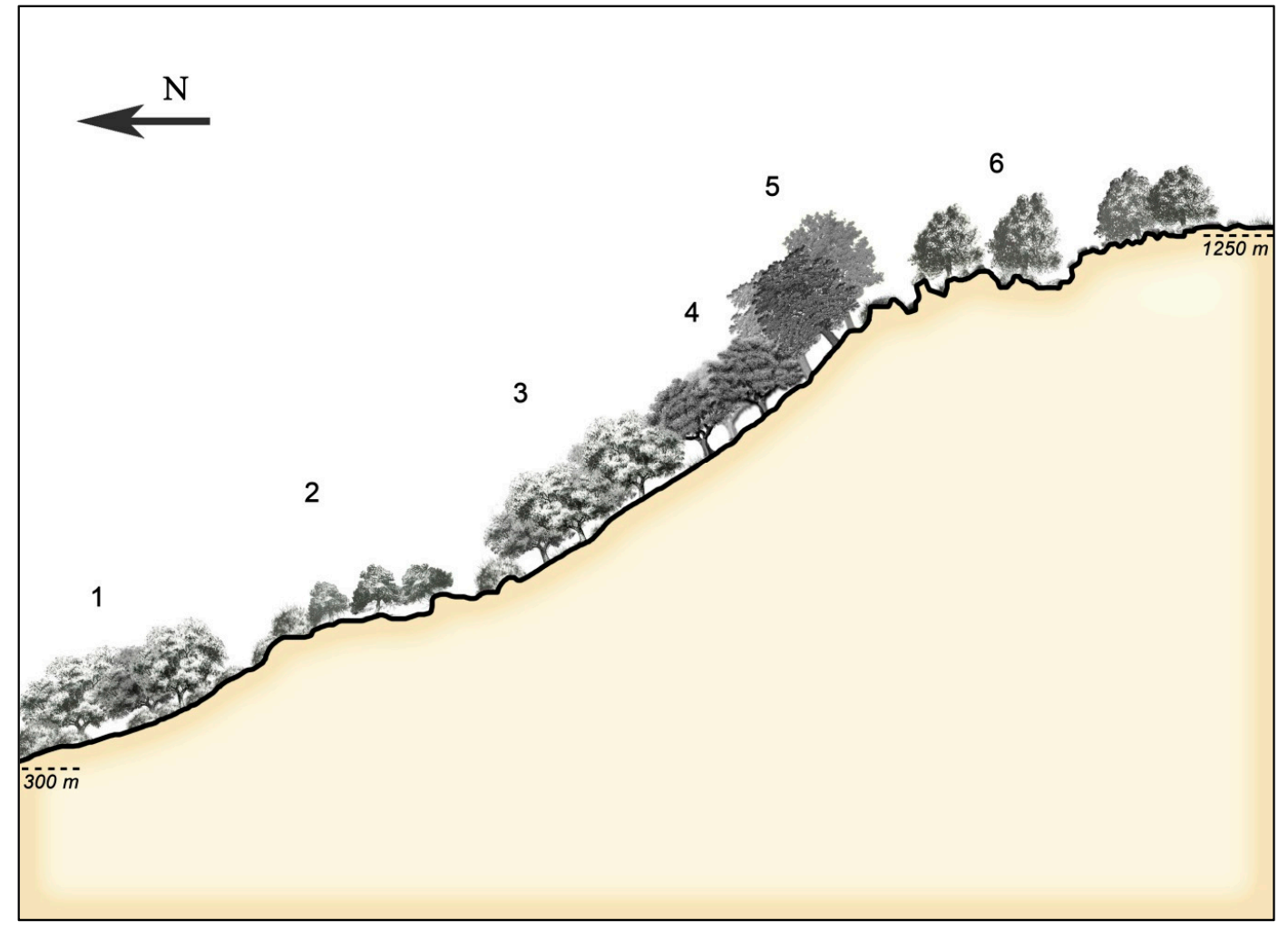

Figure 6. 1. Myrto communis-Quercetum rotundifoliae. 2. Myrto communis-Juniperetum badiae. 3. Pyro bourgaeanae-Querctum rotundifoliae. 4. Poterio agrimonioidis-Quercetum suberis. 5. Arbuto unedonis-Quercetum pyrenaicae. 6. Echinosparto iberici-Juniperetum badiae. 


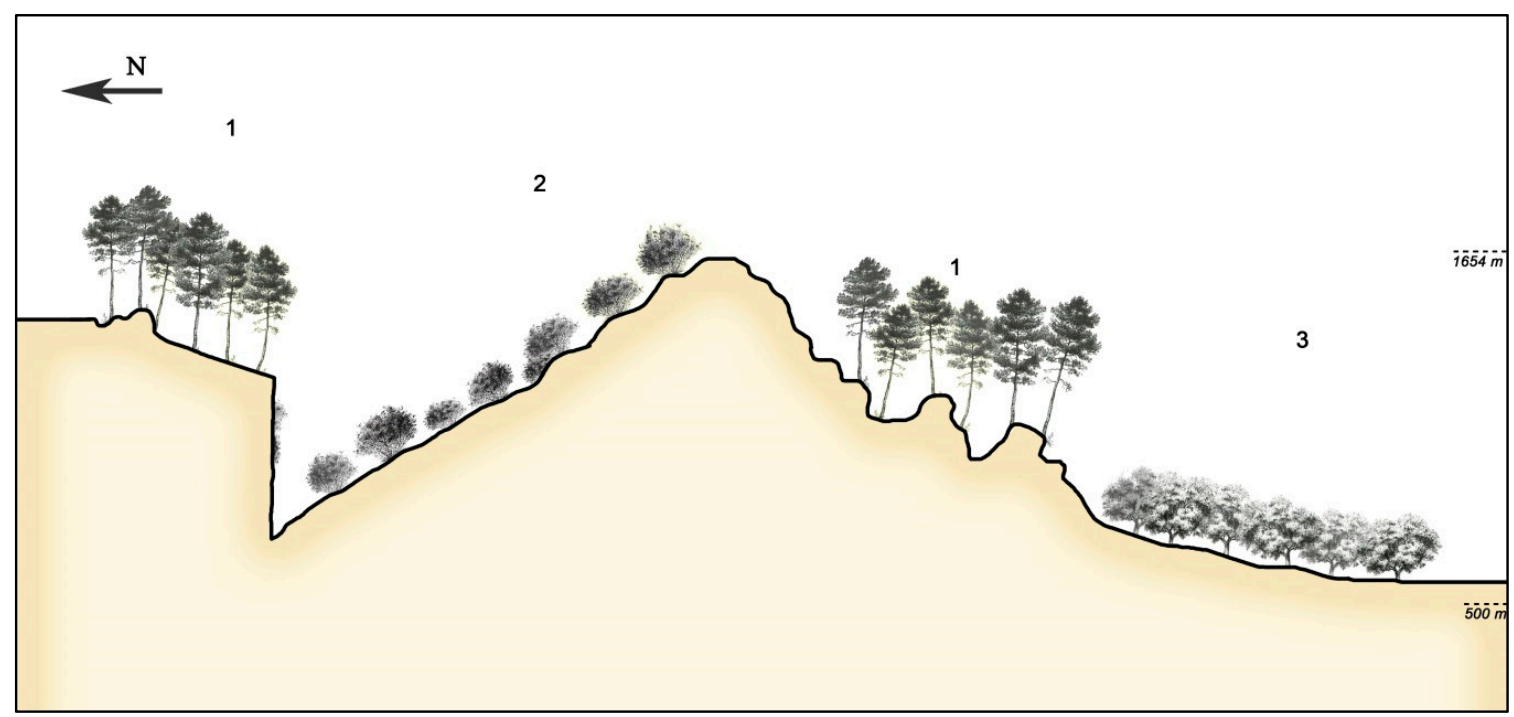

Figure 7. 1) Pinewood of Rhamno lycioidis-Pinetum halepensis. 2) Communities of Teline patentis-Pistacietum terebinthi. 3) Holm oak forest of Paeonio-Quercetum rotundifoliae (Cazorla, Mágina).

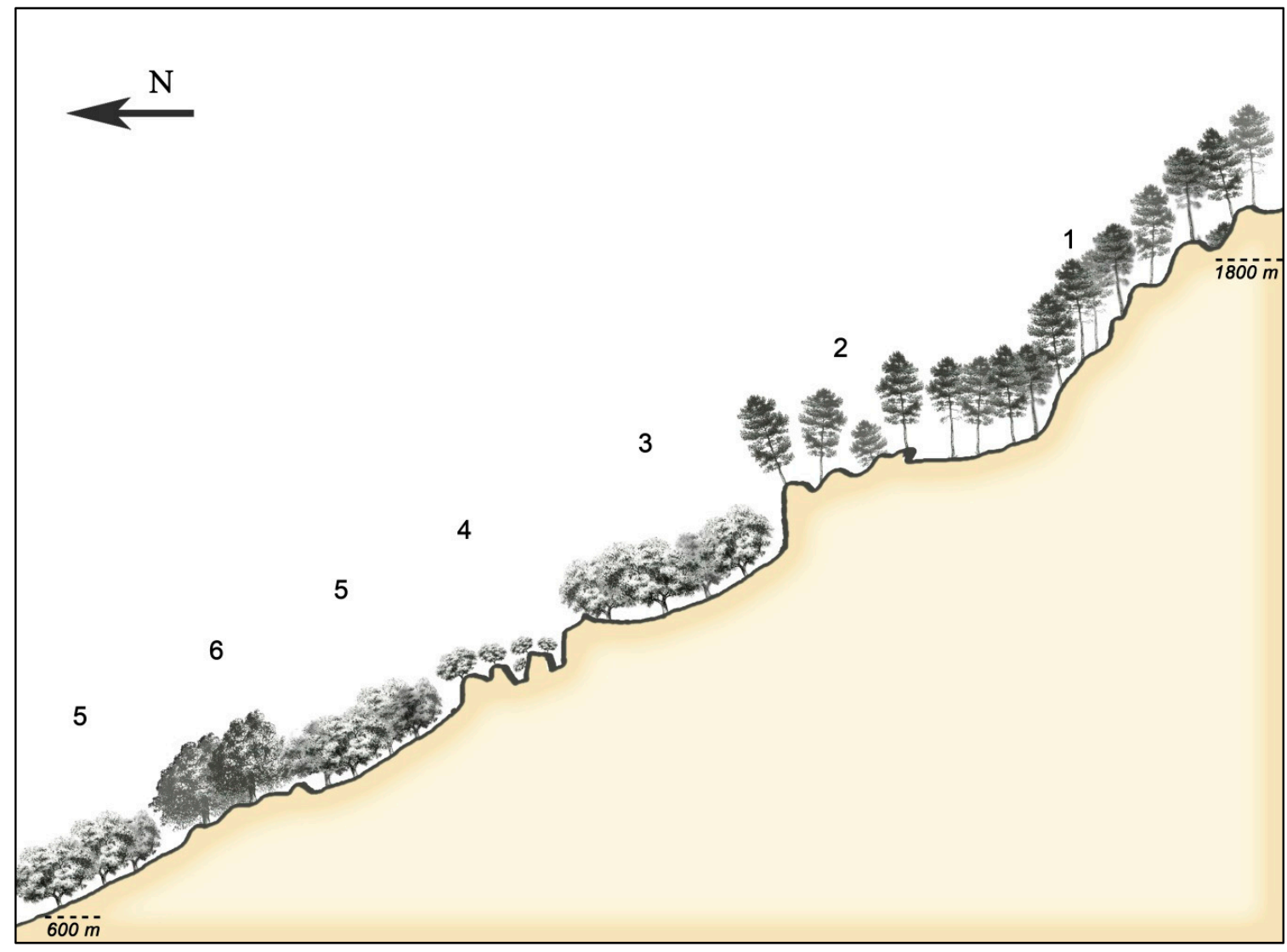

Figure 8. 1) and 2) Pine forest of Junipero phoeniceae-Pinetum clusianae. 3) Forests of Viburno tini-Quercetum alpestris, Berberido hispanicae-Quercetum alpestris, and Daphno latifoliae-Aceretum granatensi. 4) Edaphoxerophilous holm oak forest of Junipero phoeniceae-Quercetum rotundifoliae. 5) Holm oak forest of Paeonio coriaceae-Quercetum rotundifoliae. 6) Holm oak and juniper forest of Juniperetum phoeniceae-badiae (Cazorla). 


\section{Conclusions}

The different groups of communities proposed for the Luso-Extremaduran province occupy areas whose dominant ecological factor is the xericity of the substrate. These are edaphoxerophilous formations with a permanent character that occupy restricted areas, but are currently in expansion, as frequent fires and the deforestation and clearing of the scrub layer have led to an extension of eroded areas due to soil loss: indeed, the absence or presence of fire is the main agent of vegetation change in areas with low anthropic influence [55]. These biotopes do not tend to be occupied by Fagaceae, and the forests of Quercus ilex subsp. ballota are relegated to less inhospitable territories. If the factors that condition this dynamic persist, we will continue to see an exponential rise in the area occupied by species from the genus Juniperus. We can therefore predict a change in the landscape in the future, with a strong predominance of gymnosperms over angiosperms, as the former are better adapted to extreme conditions. These areas in expansion do not present a serious threat unless there is excessive pressure from livestock farming, which could lead to an alteration in these habitats that are rich in endemic species; endemics have a rate of 12 and account for $60 \%$ of their flora. As a result of the study of these wild areas we have detected a total of ten plant associations, of which we propose four as new. In all cases, these are Sites of Community Interest (SCI), as they include habitats with a high richness in endemic species. The recommendation is thus to implement conservation measures by applying a protection status to allow control over the management of the territory, for example by establishing micro-reserves for the conservation of flora and habitats, according to Spampinato et al. [56].

Soil water in semiarid areas plays an important role in evapotranspiration [57]. The significance of evapotranspiration is confirmed as a tool for improving our understanding of environmental changes, and according to Liu et al. [58], has a direct connection with society. Another important aspect is that the phytosociological approach and statistical analysis are fundamental for the study and greater knowledge of plant communities; several authors consider all these data (plus others such as phytotoponims) to be crucial to their conservation and/or restoration [20,56,59-63]. It is worth noting that vegetation is a key element for society in general and for communities that share neighbouring geographical territories. The increase in pollution, and particularly in $\mathrm{CO}_{2}$ emissions, can be mitigated by the absorption of this greenhouse gas by plants that convert it into organic matter [64]. In many cases. this organic matter can be used in a variety of forms for building the cities of the future, i.e., the cork of Quercus suber L. [65-69]. Forest management is known to be a good tool for removing atmospheric $\mathrm{CO}_{2}$ [70]. In particular, this study highlights the importance of planning actions for the efficient management of Habitat 5210 "Arborescent matorral with Juniperus ssp." present in Portugal and Spain. In fact, this kind of habitat-among others-could also sequester a substantial amount of $\mathrm{CO}_{2}$, as in the case of the juniper forest in Central Spain, which is characterised by other species of Juniperus such as J. thurifera L. and J. communis L. [71]. It is today essential to develop a modern forest management system in protected areas, and to promote the importance of forests and their extensions as a means of protecting and improving the natural environment, according to Rădulescu et al. [72]. In view of this, and since vegetation knows no political borders, it is desirable to plan actions involving cross-border-cooperation projects based on other experiences in this field to ensure sustainable development and territorial cohesion between Spain and Portugal [73,74]. 


\section{Syntaxonomical scheme}

QUERCETEA ILICIS Br.-Bl. ex A. O. Bolòs 1950

Pistacio lentisci-Rhamnetalia alaterni Rivas-Martínez 1975

Juniperion badiae Cano, Rodríguez Torres, Pinto Gomes, García Fuentes, Torres, Salazar,

Ruiz, Cano-Ortiz \& Montilla 2007 ex Mucina et al. 2016 nom. corr. hoc loco

Festuco merinoi-Juniperetum badiae (Rivas-Martínez \& Sánchez Mata 1989)

Sánchez Mata 1999 corr. Rivas-Martínez \& Sánchez Mata 2011 nom. corr.

hoc loco

Cytiso tribracteolati-Juniperetum oxycedri Pérez Latorre, Galán \& Cabezudo in

Pérez Latorre, Galán, Navas P., Gil \& Cabezudo 1999

Echinosparto iberici-Juniperetum badiae Rodríguez Torres \& Cano in Cano,

Rodríguez Torres, Pinto Gomes, García Fuentes, Torres, Salazar, Ruiz,

Cano-Ortiz \& Montilla 2007 nom. corr. hoc loco

Cytiso eriocarpi-Juniperetum badiae Pinto \& Cano in Cano, Rodríguez Torres,

Pinto Gomes, García Fuentes, Torres, Salazar, Ruiz, Cano-Ortiz \& Montilla 2007 nom. corr. hoc loco

Pistacio terebinthi-Juniperetum badiae Cano, Rodríguez Torres, Pinto Gomes,

García, Torres, Salazar, Ruiz, Cano-Ortiz \& Montilla 2007 nom. corr. hoc loco

Stipo tenacissimae-Juniperetum badiae Cano, Rodríguez Torres, Pinto Gomes,

García Fuentes, Torres, Salazar, Ruiz, Cano-Ortiz \& Montilla 2007 nom. corr.

hoc loco

Juniperetum phoeniceae-badiae ass. nova hoc loco

Teline patentis-Pistacietum terebinthi ass. nova hoc loco

Myrto communis-Juniperetum badiae ass. nova hoc loco

Genisto polyanthi-Juniperetum badiae ass. nova hoc loco

Author Contributions: Conceptualization: E.C.; data curation: C.M.M., A.C.-O., S.D.R.G. and G.S.; formal analysis: C.M.M., A.C.-O., J.C.P.F. and S.D.R.G.; research: E.C., C.M.M., A.C.-O., J.C.P.F., A.R.T., C.J.P.G. and R.Q.-C.; methodology: E.C.; software: A.C.-O., J.C.P.F. and S.D.R.G.; supervision: E.C. and C.M.M.; writing (original draft): E.C., C.M.M. and A.C.-O.; writing (review and editing): E.C., C.M.M., A.C.-O., J.C.P.F., A.R.T., S.D.R.G., C.J.P.G., R.Q.-C. and G.S.

Funding: This research received no external funding.

Acknowledgments: Pru Brooke Turner (MA Cantab.) for the English translation of this article, the architect Francisco Javier Quiros Higueras for developing the vegetation profiles, and the anonymous reviewers that improved our manuscript with their comments.

Conflicts of Interest: The authors declare no conflict of interest.

\section{Appendix A}

Synthetic table of relevés: Juniperetum phoeniceae-badiae (JPB) nova; Teline patentis-Pistacietum terebinthi (TP) nova; Myrto communis-Juniperetum badiae (MJ) nova; Echinosparto iberici-Juniperetum badiae (Cano et al. 2007) (EJ) nom. corr. hoc loco; Festuco merinoi-Juniperetum badiae (Rivas-Martínez \& Sánchez-Mata in Sánchez-Mata 1989) (FJ) nom. corr. hoc loco; Cytiso eriocarpi-Juniperetum badiae (Pinto \& Cano in Cano et al. 2007) (CJ) nom. corr. hoc loco; Pistacio terebinthi-Juniperetum badiae (Rodríguez Torres \& Cano in Cano et al. 2007) (PJ) nom. corr. hoc loco; Stipo tenacissimae-Juniperetum badiae (Cano et al. 2007) (SJ) nom. corr. hoc loco; Genisto polyanthi-Juniperetum badiae (GJ) nova. 
Table A1. Synthetic table of relevés.

\begin{tabular}{|c|c|c|c|c|c|c|c|c|c|}
\hline Characteristic & JPB & TP & MJ & EJ & FJ & CJ & PJ & SJ & GJ \\
\hline Juniperus oxycedrus subsp. badia & V & V & $\mathrm{V}$ & V & V & V & $\mathrm{V}$ & V & $\mathrm{V}$ \\
\hline Quercus rotundifolia & IV & IV & III & III & $\mathrm{V}$ & - & V & $\mathrm{V}$ & $\mathrm{V}$ \\
\hline Asparagus acutifolius & I & I & I & - & - & - & II & II & I \\
\hline Pistacia lentiscus & I & III & II & - & - & III & - & - & I \\
\hline $\begin{array}{l}\text { Juniperus oxycedrus subsp. } \\
\text { oxycedrus }\end{array}$ & IV & - & I & - & - & $\mathrm{V}$ & I & - & I \\
\hline Quercus coccifera & I & III & I & - & - & - & $\mathrm{I}$ & III & - \\
\hline Daphne gnidium & III & - & I & - & - & II & $\mathrm{V}$ & III & - \\
\hline Thapsia villosa & I & I & I & - & - & - & III & $\mathrm{I}$ & - \\
\hline Pistacia terebinthus & I & $\mathrm{V}$ & II & - & - & I & IV & - & - \\
\hline Jasminum fruticans & I & II & $\mathrm{I}$ & - & - & - & III & - & - \\
\hline Quercus faginea & I & I & - & - & - & - & I & - & - \\
\hline Rubia peregrina & $\mathrm{I}$ & $\mathrm{I}$ & $\mathrm{I}$ & - & - & - & I & - & - \\
\hline Phillyrea latifolia & I & I & I & - & - & $\mathrm{I}$ & - & - & - \\
\hline Rhamnus alaternus & $\mathrm{I}$ & I & I & - & - & $\mathrm{I}$ & - & - & - \\
\hline Smilax aspera & $\mathrm{I}$ & I & I & - & - & - & - & - & - \\
\hline Carex hallerana & $\mathrm{I}$ & - & - & - & - & - & - & $\mathrm{I}$ & - \\
\hline Juniperus phoenicea & $\mathrm{V}$ & $\mathrm{I}$ & - & - & - & - & - & - & - \\
\hline Crataegus laciniata & I & I & - & - & - & - & - & - & - \\
\hline Paeonia broteroi & I & - & - & - & - & - & - & - & - \\
\hline Pinus halepensis & I & - & - & - & - & - & - & - & - \\
\hline Teline patens & - & III & - & - & - & - & - & - & - \\
\hline Rhamnus myrtifolius & - & $\mathrm{I}$ & - & - & - & - & - & - & - \\
\hline Coronilla glauca & - & I & - & - & - & - & - & - & - \\
\hline Phillyrea angustifolia & - & I & III & I & - & $\mathrm{V}$ & - & - & I \\
\hline Olea europea var. sylvestris & - & I & II & - & - & II & II & I & - \\
\hline Viburnum tinus & - & I & $\mathrm{I}$ & - & - & $\mathrm{I}$ & - & - & - \\
\hline Arbutus unedo & - & II & II & $\mathrm{I}$ & - & - & - & - & - \\
\hline Ruscus aculeatus & - & I & - & - & - & $\mathrm{I}$ & - & - & - \\
\hline Pinus pinaster & - & I & - & $\mathrm{I}$ & - & - & - & - & - \\
\hline Erica arborea & - & - & I & $\mathrm{I}$ & IV & $\mathrm{V}$ & - & - & $\mathrm{I}$ \\
\hline Rhamnus lycioides & - & - & I & - & - & - & II & $\mathrm{I}$ & - \\
\hline Osyris alba & - & - & $\mathrm{I}$ & - & - & - & III & $\mathrm{I}$ & - \\
\hline Quercus suber & - & - & $\mathrm{I}$ & $\mathrm{I}$ & - & - & $\mathrm{I}$ & - & - \\
\hline Quercus broteroi & - & - & $\mathrm{I}$ & - & - & - & I & - & - \\
\hline Pyrus bourgaeana & - & - & I & - & - & - & I & - & - \\
\hline Myrtus communis & - & - & II & - & - & III & - & - & - \\
\hline Asparagus aphyllus & - & - & I & - & - & $\mathrm{I}$ & - & - & - \\
\hline Rhamnus oleoides & - & - & I & - & - & II & - & - & - \\
\hline Pistacia $x$ saportae & - & - & I & - & - & $\mathrm{I}$ & - & - & - \\
\hline Asparagus albus & - & - & $\mathrm{I}$ & - & - & - & - & - & - \\
\hline Teucrium fruticans & - & - & I & - & - & - & - & - & - \\
\hline Quercus marianica & - & - & I & - & - & - & - & - & - \\
\hline Quercus canariensis & - & - & $\mathrm{I}$ & - & - & - & - & - & - \\
\hline Phlomis purpurea & - & - & $\mathrm{I}$ & - & - & - & - & - & - \\
\hline Crataegus monogyna & - & - & $\mathrm{I}$ & - & - & - & - & - & - \\
\hline Genista polyanthos & - & - & - & - & - & - & - & - & $\mathrm{V}$ \\
\hline \multicolumn{10}{|l|}{ Companions } \\
\hline Urginea maritima & $\mathrm{I}$ & $\mathrm{I}$ & II & I & - & - & I & I & I \\
\hline Thymus mastichina & II & I & I & - & - & - & V & II & I \\
\hline Rosmarinus officinalis & III & III & III & - & - & - & I & - & II \\
\hline Asphodelus albus & III & I & $\mathrm{I}$ & II & - & - & - & - & II \\
\hline
\end{tabular}


Table A1. Cont.

\begin{tabular}{|c|c|c|c|c|c|c|c|c|c|}
\hline Characteristic & JPB & TP & MJ & EJ & FJ & CJ & PJ & SJ & GJ \\
\hline Cistus albidus & III & III & II & - & - & - & - & III & I \\
\hline Dactylis hispanica & I & I & $\mathrm{I}$ & - & IV & - & $\mathrm{I}$ & IV & - \\
\hline Retama sphaerocarpa & $\mathrm{I}$ & - & $\mathrm{I}$ & - & - & - & $\mathrm{V}$ & II & I \\
\hline Asplenium ceterach & I & I & I & - & - & - & $\mathrm{I}$ & I & - \\
\hline Rubus ulmifolius & I & II & III & - & - & - & $\mathrm{I}$ & - & - \\
\hline Phagnalon saxatile & $\mathrm{I}$ & - & - & - & - & - & I & III & - \\
\hline Geranium purpureum & $\mathrm{I}$ & $\mathrm{I}$ & - & - & - & - & - & - & I \\
\hline Phlomis lychnitis & II & I & - & - & - & - & - & III & - \\
\hline $\begin{array}{l}\text { Lonicera periclymenum subsp. } \\
\text { hispanica }\end{array}$ & $\mathrm{I}$ & I & - & - & - & - & $\mathrm{I}$ & - & - \\
\hline Staehelina dubia & $\mathrm{I}$ & I & - & - & - & - & - & I & - \\
\hline Santolina canescens & I & - & - & - & - & - & I & - & - \\
\hline Halimium atriplicifolium & $\mathrm{I}$ & $\mathrm{I}$ & $\mathrm{I}$ & - & - & - & - & - & - \\
\hline Melica minuta & $\mathrm{I}$ & I & $\mathrm{I}$ & - & - & - & - & - & - \\
\hline Sanguisorba minor & $\mathrm{I}$ & - & $\mathrm{I}$ & - & - & - & - & - & - \\
\hline Thymus zygis subsp. gracilis & I & I & - & - & - & - & - & - & - \\
\hline Thymus orospedanus & III & II & - & - & - & - & - & - & - \\
\hline Aphyllantes monspeliensis & I & II & - & - & - & - & - & - & - \\
\hline Rosa canina & I & I & - & - & - & - & - & - & - \\
\hline Brachypodium retusum & II & I & - & - & - & - & - & - & - \\
\hline Hedera ibernica & I & I & - & - & - & - & - & - & - \\
\hline Helleborus foetidus & $\mathrm{I}$ & I & - & - & - & - & - & - & - \\
\hline Lavandula latifolia & $\mathrm{I}$ & - & - & - & - & - & - & - & - \\
\hline Cistus monspeliensis & $\mathrm{I}$ & - & - & - & - & - & - & - & - \\
\hline Teucrium capitatum & I & - & - & - & - & - & - & - & - \\
\hline Teucrium rotundifolium & $\mathrm{I}$ & - & - & - & - & - & - & - & - \\
\hline Helianthemum ledifolium & I & - & - & - & - & - & - & - & - \\
\hline Clematis flammula & I & - & - & - & - & - & - & - & - \\
\hline Linum suffruticosum & $\mathrm{I}$ & - & - & - & - & - & - & - & - \\
\hline Bupleurum rigidum & I & - & - & - & - & - & - & - & - \\
\hline Ilex aquifolium & I & - & - & - & - & - & - & - & - \\
\hline Fumana thymifolia & $\mathrm{I}$ & - & - & - & - & - & - & - & - \\
\hline Lonicera splendida & I & - & - & - & - & - & - & - & - \\
\hline Leuzea conifera & $\mathrm{I}$ & - & - & - & - & - & - & - & - \\
\hline Berberis hispanica & $\mathrm{I}$ & - & - & - & - & - & - & - & - \\
\hline Buxus sempervirens & I & - & - & - & - & - & - & - & - \\
\hline Pinus salzmannii & I & - & - & - & - & - & - & - & - \\
\hline Helianthemum syriacum & I & - & - & - & - & - & - & - & - \\
\hline Filipendula vulgaris & $\mathrm{I}$ & - & - & - & - & - & - & - & - \\
\hline Polygala monspeliaca & $\mathrm{I}$ & - & - & - & - & - & - & - & - \\
\hline Ophrys tenthrediniferfa & $\mathrm{I}$ & - & - & - & - & - & - & - & - \\
\hline $\begin{array}{l}\text { Helianthemum cinereum subsp. } \\
\text { rotundifolium }\end{array}$ & I & - & - & - & - & - & - & - & - \\
\hline Ptilostemon hispanicus & $\mathrm{I}$ & - & - & - & - & - & - & - & - \\
\hline Anthyllis vulneraria subsp. maura & $\mathrm{I}$ & - & - & - & - & - & - & - & - \\
\hline Arrhenatherum album & $\mathrm{I}$ & - & - & - & - & - & - & - & - \\
\hline Erinacea anthyllis & I & - & - & - & - & - & - & - & - \\
\hline
\end{tabular}


Table A1. Cont.

\begin{tabular}{|c|c|c|c|c|c|c|c|c|c|}
\hline Characteristic & JPB & TP & MJ & EJ & FJ & CJ & PJ & SJ & GJ \\
\hline Fumana paradoxa & I & - & - & - & - & - & - & - & - \\
\hline Helictotrichon filifolium & I & - & - & - & - & - & - & - & - \\
\hline Crupina crupinastrum & I & - & - & - & - & - & - & - & - \\
\hline Narcissus assoanus & I & - & - & - & - & - & - & - & - \\
\hline Medicago rigidula & I & - & - & - & - & - & - & - & - \\
\hline Genista scorpius & $\mathrm{I}$ & - & - & - & - & - & - & - & - \\
\hline Crocus serotinus subsp. salzmannii & I & - & - & - & - & - & - & - & - \\
\hline Prunus spinosa & I & - & - & - & - & - & - & - & - \\
\hline Cytisus scoparius subsp. reverchonii & I & - & - & - & - & - & - & - & - \\
\hline Fumana laevipes & I & - & - & - & - & - & - & - & - \\
\hline Genista boissieri & I & - & - & - & - & - & - & - & - \\
\hline Amelanchier ovalis & I & - & - & - & - & - & - & - & - \\
\hline Muscari giennense & I & - & - & - & - & - & - & - & - \\
\hline Thymus granatensis & I & - & - & - & - & - & - & - & - \\
\hline Biscutella sempervirens & I & - & - & - & - & - & - & - & - \\
\hline Sedum brevifolium & - & I & $\mathrm{I}$ & $\mathrm{I}$ & - & IV & - & $\mathrm{I}$ & II \\
\hline Mucizonia hispida & - & I & $\mathrm{I}$ & I & - & - & - & - & II \\
\hline Tamus communis & - & II & $\mathrm{I}$ & - & - & - & II & - & - \\
\hline Fraxinus angustifolia & - & I & $\mathrm{I}$ & - & - & - & - & - & I \\
\hline Polygala rupestris & - & I & I & - & - & - & - & - & - \\
\hline Sedum sediforme & - & I & I & - & - & - & - & - & - \\
\hline Vitis vinifera subsp. sylvestris & - & I & I & - & - & - & - & - & - \\
\hline Lithodora fruticosa & - & I & - & - & - & - & - & - & - \\
\hline Biscutella valentina & - & I & - & - & - & - & - & - & - \\
\hline Rubus caesius & - & I & - & - & - & - & - & - & - \\
\hline Clematis vitalba & - & I & - & - & - & - & - & - & - \\
\hline Euphorbia characias & - & $\mathrm{I}$ & - & - & - & - & - & - & - \\
\hline Genista cinerea subsp. speciosa & - & I & - & - & - & - & - & - & - \\
\hline Dianthus lusitanus & - & - & II & III & IV & IV & I & II & IV \\
\hline Cistus ladanifer & - & - & III & III & - & III & I & I & III \\
\hline Halimium umbellatum subsp. viscosum & & - & I & $\mathrm{I}$ & - & III & $\mathrm{I}$ & I & $\mathrm{I}$ \\
\hline Arrhenatherum bulbosum & - & - & I & $\mathrm{I}$ & - & II & II & - & III \\
\hline Halimium ocymoides & - & - & I & II & - & III & - & - & II \\
\hline Lavandula sampaiana & - & - & II & II & - & III & IV & III & - \\
\hline Sedum dasyphyllum & - & - & I & $\mathrm{I}$ & - & - & - & - & $\mathrm{I}$ \\
\hline Coincya longirrostra & - & - & I & I & - & - & - & - & $\mathrm{I}$ \\
\hline Digitalis mariana & - & - & $\mathrm{I}$ & II & - & - & - & - & $\mathrm{I}$ \\
\hline Jasione mariana & - & - & I & II & - & - & - & - & III \\
\hline Linaria saxatilis & - & - & $\mathrm{I}$ & II & - & - & - & - & II \\
\hline Lavandula luisieri & - & - & I & I & - & - & - & - & I \\
\hline Elymus caninus & - & - & $\mathrm{I}$ & $\mathrm{I}$ & - & - & - & - & $\mathrm{I}$ \\
\hline Nerium oleander & - & - & $\mathrm{I}$ & - & - & - & - & - & $\mathrm{I}$ \\
\hline Anogramma leptophylla & - & - & I & - & - & - & - & - & I \\
\hline Adenocarpus telonensis & - & - & II & - & - & - & I & $\mathrm{I}$ & - \\
\hline Cytisuss striatus subsp. eriocarpus & - & - & I & - & $\mathrm{V}$ & IV & I & - & - \\
\hline Cistus salvifolius & - & - & I & - & - & III & - & III & - \\
\hline Bryonia cretica & - & - & $\mathrm{I}$ & - & - & - & I & - & - \\
\hline
\end{tabular}


Table A1. Cont.

\begin{tabular}{|c|c|c|c|c|c|c|c|c|c|}
\hline Characteristic & JPB & TP & MJ & EJ & FJ & CJ & PJ & SJ & GJ \\
\hline Flueggea tinctoria & - & - & $\mathrm{I}$ & - & - & - & - & - & I \\
\hline Genista hirsuta & - & - & I & - & - & - & $\mathrm{I}$ & - & - \\
\hline Teucrium gnaphalodes & - & - & I & - & - & - & - & I & - \\
\hline Teucrium pseudochamaepytis & - & - & I & - & - & - & - & III & - \\
\hline Cheilanthes tinaei & - & - & I & - & - & - & - & I & - \\
\hline Calluna vulgaris & - & - & $\mathrm{I}$ & $\mathrm{I}$ & - & II & - & - & - \\
\hline Cheilanthes hispanica & - & - & $\mathrm{I}$ & - & - & II & - & - & - \\
\hline Adenocarpus argyrophyllus & - & - & $\mathrm{I}$ & II & - & - & - & - & - \\
\hline Ericca scoparia & - & - & I & I & - & - & - & - & - \\
\hline Astragalus lusitanicus & - & - & $\mathrm{I}$ & I & - & - & - & - & - \\
\hline Jasione tomentosa & - & - & I & $\mathrm{I}$ & - & - & - & - & - \\
\hline Cheilanthes maderensis & - & - & II & - & - & - & - & - & - \\
\hline Arisarum simorhinum & - & - & $\mathrm{I}$ & - & - & - & - & - & - \\
\hline Sedum album & - & - & I & - & - & - & - & - & - \\
\hline Stipa capensis & - & - & $\mathrm{I}$ & - & - & - & - & - & - \\
\hline Hyacinthoides hispanica & - & - & $\mathrm{I}$ & - & - & - & - & - & - \\
\hline Pterocepalus diandrus & - & - & I & - & - & - & - & - & - \\
\hline Digitalis heywoodii var. albicans & - & - & $\mathrm{I}$ & - & - & - & - & - & - \\
\hline Antirrhinum graniticum subsp. onube & nsis & - & $\mathrm{I}$ & - & - & - & - & - & - \\
\hline Cytisus scoparius subsp. bourgaei & - & - & $\mathrm{I}$ & - & - & - & - & - & - \\
\hline Aristolochia baetica & - & - & I & - & - & - & - & - & - \\
\hline Micromeria graeca & - & - & I & - & - & - & - & - & - \\
\hline Dianthus crassipes & - & - & $\mathrm{I}$ & - & - & - & - & - & - \\
\hline Jasonia glutinosa & - & - & I & - & - & - & - & - & - \\
\hline Helianthemum croceum & - & - & $\mathrm{I}$ & - & - & - & - & - & - \\
\hline Stipa gigantea & - & - & - & II & - & - & I & II & II \\
\hline Conopodium capillifolium & - & - & - & I & - & I & - & - & I \\
\hline Festuca elegans & - & - & - & II & $\mathrm{V}$ & - & - & - & $\mathrm{I}$ \\
\hline Armeria capitella & - & - & - & I & - & - & - & - & I \\
\hline Narcissus rupicola & - & - & - & I & - & - & - & - & I \\
\hline Digitalis thapsi & - & - & - & I & - & II & II & - & - \\
\hline Lavandula pedunculata & - & - & - & - & IV & - & I & - & - \\
\hline Erica australis & - & - & - & $\mathrm{I}$ & - & II & - & - & - \\
\hline Gladiolus illyricus & - & - & - & $\mathrm{I}$ & - & I & - & - & - \\
\hline Echinospartum ibericum & - & - & - & II & - & - & - & - & - \\
\hline Quercus pyrenaica & - & - & - & $\mathrm{I}$ & - & - & - & - & - \\
\hline Arenaria querioides & - & - & - & $\mathrm{I}$ & - & - & - & - & - \\
\hline Armeria arenaria subsp. segoviensis & - & - & - & I & - & - & - & - & - \\
\hline Erica scoparia & - & - & - & $\mathrm{I}$ & - & - & - & - & - \\
\hline Leucanthemopsis flaveola & - & - & - & $\mathrm{I}$ & - & - & - & - & - \\
\hline Festuca summilusitanica & - & - & - & - & V & - & - & - & - \\
\hline Cytisus oromediterraneus & - & - & - & - & IV & - & - & - & - \\
\hline Juniperus hemisphaerica & - & - & - & - & IV & - & - & - & - \\
\hline Arenaria grandiflora & - & - & - & - & $\mathrm{V}$ & - & - & - & - \\
\hline Thymus $x$ bractichina & - & - & - & - & $\mathrm{V}$ & - & - & - & - \\
\hline Pteridium aquilinum & - & - & - & - & V & - & - & - & - \\
\hline Sorbus aucuparia & - & - & - & - & II & - & - & - & - \\
\hline Jasione sessiliflora & - & - & - & - & IV & - & - & - & - \\
\hline
\end{tabular}


Table A1. Cont.

\begin{tabular}{lccccccccc}
\hline \multicolumn{1}{c}{ Characteristic } & JPB & TP & MJ & EJ & FJ & CJ & PJ & SJ & GJ \\
\hline Genista cinerascens & - & - & - & - & I & - & - & - & - \\
\hline Sedum hirsutum & - & - & - & - & IV & IV & - & - & - \\
\hline Simethis planifolia & - & - & - & - & - & II & - & - & - \\
\hline Polypodium cambricum & - & - & - & - & - & I & - & - & - \\
\hline Adenocarpus anisochilus & - & - & - & - & - & I & - & - & - \\
\hline Hypericum linearifolium & - & - & - & - & - & I & - & - & - \\
\hline Thymus zygis & - & - & - & - & - & - & I & III & - \\
\hline Helichrysum stoechas & - & - & - & - & - & - & I & IV & - \\
\hline Cynosurus echinatus & - & - & - & - & - & - & I & I & - \\
\hline Santolina rosmarinifolia & - & - & - & - & - & - & I & I & - \\
\hline Hyparrhenia hirta & - & - & - & - & - & - & I & I & - \\
\hline Cytisus scoparius subsp. scoparius & - & - & - & - & - & - & III & - & - \\
\hline Helianthemum appeninum & - & - & - & - & - & - & I & - & - \\
\hline Hedera helix & - & - & - & - & - & - & I & - & - \\
\hline Lonicera implexa & - & - & - & - & - & - & I & - & - \\
\hline Thapsia maxima & - & - & - & - & - & - & I & - & - \\
\hline Antirrhinum graniticum & - & - & - & - & - & - & I & - & - \\
\hline Stipa tenacissima & - & - & - & - & - & - & - & III & - \\
\hline Cytisus multiflorus & - & - & - & - & - & - & - & I & - \\
\hline Cistus populifolius $x$ C. salvifolius & - & - & - & - & - & - & - & - & I \\
\hline Festuca rothmaleri & - & - & - & - & - & - & - & - & I \\
\hline Conopodium bourgaei & - & - & - & - & - & - & - & - & I \\
\hline Asplenium billotii & - & - & - & - & - & - & - & - & I \\
\hline Scrophularia canina & - & - & - & - & - & - & - & - & I \\
\hline Genista florida & - & - & - & - & - & - & - & - & I \\
\hline
\end{tabular}

The values from $\mathrm{I}$ to $\mathrm{V}$ represent the degree of presence of a species in an association. $\mathrm{V}=$ presence between 80 and 100\%; IV = between 60 and $80 \%$; III = between 40 and $60 \%$; II = between 20 and $40 \%$; I = <20\%; "-" indicates its absence [75]. The floristic groups differentiating the plant associations are highlighted in grey.

\section{References}

1. Adams, R.P. Taxonomy of Juniperus, section Juniperus: Sequence analysis of nrDNA and five cpDNA regions. Phytologia 2012, 94, 280-297.

2. Adams, R.P. Junipers of the World: The Genus Juniperus, 4th ed.; Trafford Publishing: Vancouver, Canada, 2014.

3. Adams, R.P. Morphological comparison and key to Juniperus deltoides and J. oxycedrus. Phytologia 2014, 96, 58-62.

4. Amaral Franco, J. Juniperus. In Flora Ibérica. Plantas Vasculares de la Península Ibérica e Islas Baleares (Vol. 1); Castroviejo, S., Laínz, M., López González, G., Monserrat, P., Muñoz Garmendia, F., Paiva, J., Villar, L., Eds.; Real Jardín Botánico, CSIC: Madrid, Spain, 1986; pp. 181-188.

5. Pignatti, S. Juniperus; Flora d'Italia 1; Edagricole: Bologna, Italy, 1982.

6. Brus, R.; Idzjtic', M.; Jarni, K. Morphologic variation in northern marginal Juniperus oxycedrus L. subsp. oxycedus population in Istria. Plant Biosyst. 2016, 150, 274-284. [CrossRef]

7. Adams, R.P. Juniperus deltoides, a new species, and nomenclatural notes on Juniperus polycarpos and Juniperus turcomanica (Cupressaceae). Phytologia 2004, 86, 49-53.

8. Adams, R.P.; Tashev, A.N. Geographical variation in leaf oils of Juniperus deltoides from Bulgaria, Greece, Italy and Turkey. Phytologia 2012, 94, 310-318.

9. Adams, R.P.; Farzaliyev, V.; Gucel, S.; Leschner, H.V.; Mataraci, T.; Tashev, A.N.; Schwarzbach, A.E. nrDNA and petN-psbM sequencing reveals putative Juniperus oxycedrus L. from Azerbaijan, Bulgaria, Cyprus and Israel to be J. deltoides R.P. Adams. Phytologia 2015, 97, 286-290.

10. Adams, R.P.; Joaquin Altarejos, J.; Fernandez, C.; Camacho, A. The leaf essential oils and taxonomy of Juniperus oxycedrus L. subsp. oxycedrus, subsp. badia (H. Gay) Debeaux, and subsp. macrocarpa (Sibth. \& Sm.). Ball. J. Essent. Oil Res. 1999, 11, 167-172. 
11. Salido, S.; Altarejos, J.; Nogueras, M.; Sánchez, A.; Pannecouque, C.; Witvrouw, M.; De Clercq, E. Chemical studies of essential oils of Juniperus oxycedrus ssp. badia. J. Ethnopharmacol. 2002, 81, 129-134. [CrossRef]

12. Adams, R.P. Systematics of Juniperus section Juniperus based on leaf essential oils and random amplified polymorphic DNAs (RAPDs). Biochem. Syst. Ecol. 2000, 28, 515-528. [CrossRef]

13. Adams, R.P.; Morris, J.A.; Pandey, R.N.; Schwarzbach, A.E. Cryptic speciation between Juniperus deltoides and Juniperus oxycedrus (Cupressaceae) in the Mediterranean. Biochem. Syst. Ecol. 2005, 33, 771-787. [CrossRef]

14. Roma-Marzio, F.; Najar, B.; Alessandri, J.; Pistelli, L.; Peruzzi, L. Taxonomy of prickly juniper (Juniperus oxycedrus group): A phytochemical-morphometric combined approach at the contact zone of two cryptospecies. Phytochemistry 2017, 141, 48-60. [CrossRef] [PubMed]

15. Rivas-Martínez, S. Memoria del mapa de Series de Vegetación de España; ICONA. Serie Técnica. Publ. Ministerio Agricultura, Pesca y Alimentación: Madrid, Spain, 1987; pp. 1-268.

16. Bolòs, O.; Vigo, J. Flora dels Països Catalans; Barcino: Barcelona, Spain, 1984.

17. Rivas-Martínez, S.; Díaz, T.E.; Fernández González, F.; Izco, J.; Loidi, J.; Lousa, M.; Penas, A. Vascular plant communities of Spain and Portugal. Itinera Geobot. 2002, 15, 433-922.

18. Vicioso, C. Notas sobre la flora española. An. Jardin Bot. Madrid 1946, 6, 5-89.

19. Cano, E.; Rodríguez Torres, A.; Pinto Gomes, C.J.; García Fuentes, A.; Torres, J.A.; Salazar, C.; Ruiz, L.; Cano-Ortiz, A.; Montilla, R. Analysis of the Juniperus oxycedrus L. in the centre and south of the Iberian Peninsula (Spain and Portugal). Acta Bot. Gallica 2007, 154, 79-99. [CrossRef]

20. Quinto-Canas, R.; Mendes, P.; Cano-Ortiz, A.; Musarella, C.M.; Pinto-Gomes, C. Forest fringe communities of the southwestern Iberian Peninsula. Rev. Chapingo Ser. Cie. 2018, 24, 415-434. [CrossRef]

21. Melendo, M.; Giménez, E.; Cano, E.; Gómez Mercado, F.; Valle, F. The endemic flora in the south of the Iberian Peninsula: taxonomic composition, biological spectrum, pollination, reproductive mode and dispersal. Flora 2003, 198, 260-276. [CrossRef]

22. Cano, E.; Melendo, M.; Valle, F. The plant communities of the Asplenietea trichomanis in the SW Iberian Península. Folia Geobot. 1997, 32, 361-376.

23. Cano-Ortiz, A.; Pinto Gomes, C.J.; Musarella, C.M.; Cano, E. Expansion of the Juniperus genus due to anthropic activity. In Old-Growth Forest and Coniferous Forests; Weber, R.P., Ed.; Nova Science Publishers: New York, NY, USA, 2015; pp. 55-65.

24. Mendes, P.; Meireles, C.; Vila-Viçosa, C.; Musarella, C.M.; Pinto-Gomes, C. Best management practices to face degraded territories occupied by Cistus ladanifer shrublands - Portugal case study. Plant Biosyst. 2015, 149, 494-502. [CrossRef]

25. Rivas-Martínez, S.; Loidi, J. Bioclimatology of the Iberian Peninsula. Itinera Geobot. 1999, 13, 41-47.

26. Cano, E.; Torres, J.A.; Cano-Ortiz, A.; Montilla, R.J. Una nueva asociación de matorral para la alianza Lavandulo-Genistion Boissieri en el sector subbético. Lagascalia 2005, 25, 125-133.

27. Castroviejo, S.; Laínz, M.; López González, G.; Monserrat, P.; Muñoz Garmendia, F.; Paiva, J.; Villar, L. (Eds.) Flora Ibérica. Plantas Vasculares de la Península Ibérica e Islas Baleares (Vol. 1-2); Real Jardín Botánico, CSIC: Madrid, Spain, 1990.

28. Castroviejo, S.; Aedo, C.; Cirujano, S.; Laínz, M.; Monserrat, P.; Morales, R.; Muñoz Garmendia, F.; Navarro, C.; Paiva, J.; Soriano, C. (Eds.) Flora Ibérica. Plantas Vasculares de la Península Ibérica e Islas Baleares (Vol. 3); Real Jardín Botánico, CSIC: Madrid, Spain, 1993.

29. Castroviejo, S.; Aedo, C.; Gómez Campo, C.; Laínz, M.; Monserrat, P.; Morales, R.; Muñoz Garmendia, F.; Nieto Feliner, G.; Rico, E.; Talavera, S.; Villar, L. (Eds.) Flora Ibérica. Plantas Vasculares de la Península Ibérica e Islas Baleares (Vol. 4); Real Jardín Botánico, CSIC: Madrid, Spain, 1993.

30. Castroviejo, S.; Aedo, C.; Laínz, M.; Morales, R.; Muñoz Garmendia, F.; Nieto Feliner, G.; Paiva, J. (Eds.) Flora Ibérica. Plantas Vasculares de la Península Ibérica e Islas Baleares (Vol. 5); Real Jardín Botánico, CSIC: Madrid, Spain, 1997.

31. Castroviejo, S.; Aedo, C.; Benedí, C.; Laínz, M.; Muñoz Garmendia, F.; Nieto Feliner, G.; Paiva, J. (Eds.) Flora Ibérica. Plantas Vasculares de la Península Ibérica e Islas Baleares (Vol. 8); Real Jardín Botánico, CSIC: Madrid, Spain, 1997.

32. Muñoz Garmendia, F.; Navarro, C. (Eds.) Flora Ibérica. Plantas Vasculares de la Península Ibérica e Islas Baleares (Vol. 6); Real Jardín Botánico, CSIC: Madrid, Spain, 1998. 
33. Paiva, J.; Sales, F.; Hedge, I.C.; Aedo, C.; Aldasoro, J.J.; Castroviejo, S.; Herrero, A.; Velayos, M. (Eds.) Flora Ibérica. Plantas Vasculares de la Península Ibérica e Islas Baleares (Vol. 14); Real Jardín Botánico, CSIC: Madrid, Spain, 2001.

34. Talavera, S.; Aedo, C.; Castroviejo, S.; Romero Zarco, C.; Saéz, C.; Salgueiro, F.J.; Velayos, M. (Eds.) Flora Ibérica. Plantas Vasculares de la Península Ibérica e Islas Baleares (Vol 7(1)); Real Jardín Botánico, CSIC: Madrid, Spain, 1999.

35. Talavera, S.; Aedo, C.; Castroviejo, C.; Herrero, A.; Romero Zarco, C.; Salgueiro, F.J.; Velayos, S. (Eds.) Flora Ibérica. Plantas Vasculares de la Península Ibérica e Islas Baleares (Vol. 7(2)); Real Jardín Botánico, CSIC: Madrid, Spain, 2000.

36. Tutin, T.; Heywood, V.H.; Burges, D.A.; Valentine, D.H.; Walters, S.M.; Webb, D.A. (Eds.) Flora Europaea (Vol. I to V); Cambrige at the University Press: Cambrige, UK, 1964.

37. Valdés, B.; Talavera, S.; Fernández Galiano, E. (Eds.) Flora Vascular de Andalucía Occidental; Ketres Editora S A: Barcelona, Spain, 1987.

38. Mucina, L.; Bültmann, H.; Dierßen, K.; Theurillat, J.-P.; Raus, T.; Čarni, A.; Šumberová, K.; Willner, W.; Dengler, J.; Tichý, L. Vegetation of Europe: Hierarchical floristic classification system of vascular plant, bryophyte, lichen, and algal communities. Appl. Veg. Sci. 2016, 19, 3-264. [CrossRef]

39. Sánchez Mata, D. Bioclimatología: una Ciencia Avanzada para la Caracterización del Medio Natural; Discursos de Entrada 1998; Publicaciones Institución Gran Duque de Alba: Ávila, Spain, 1999.

40. Braun-Blanquet, J. Fitosociología. Bases para el Estudio de las Comunidades Vegetales; Blume: Madrid, Spain, 1979.

41. Géhu, J.M.; Rivas-Martínez, S. Notions fondamentales de Phytosociologie. In Berichte der Internationalen Symposien der Internationalen Vereinigung für Vegetationskunde; Dierschk, H., Ed.; Cramer: Vaduz, Liechtenstein, 1981; pp. 5-33.

42. Hammer, Ø.; Harper, D.A.T.; Ryan, P.D. PAST: Paleontological Statistics Software Package for Education and Data Analysis. Palaeontologia Electronica 2001, 4, 1-9.

43. Montero Burgos, J.L.; González Rebollar, J.L. Diagramas Bioclimáticos; Ministerio de Agricultura, Pesca y Alimentación. ICONA: Madrid, Spain, 1983.

44. Pérez Latorre, A.V.; Galán de Mera, A.; Navas, P.; Navas, D.; Gil, Y. Datos sobre la flora y vegetación del Parque Natural de los Alcornocales (Cádiz-Málaga, España). Acta Bot. Malacit. 1999, 24, 133-184.

45. Pavón Núñez, M.; Hidalgo Triana, N.; Pérez Latorre, A.V. Aportaciones al conocimiento de las comunidades de Pistacia terebinthus L. y de Acer monspessulanum L. en el sur de la Península Ibérica. Lagascalia 2013, 33, 299-311.

46. Rivas-Martínez, S.; Penas, A.; Díaz-González, T.E.; Ladero-Álvarez, M.; Asensi-Marfil, A.; Díez-Garretas, B.; Molero-Mesa, J.; Valle-Tendero, F.; Cano, E.; Costa-Talens, M.; et al. Mapa de series, geoseries y geopermaseries de vegetación de España. Parte I. Itinera Geobot. 2007, 17, 4-36.

47. Rivas-Martínez, S.; Penas, A.; Díaz-González, T.E.; Ladero-Álvarez, M.; Asensi-Marfil, A.; Díez-Garretas, B.; Molero-Mesa, J.; Valle-Tendero, F.; Cano, E.; Costa-Talens, M.; et al. Mapa de series, geoseries y geopermaseries de vegetación de España. Parte II. Itinera Geobot. 2011, 18, 5-424.

48. Rivas-Martínez, S.; Penas, A.; Díaz-González, T.E.; Ladero-Álvarez, M.; Asensi-Marfil, A.; Díez-Garretas, B.; Molero-Mesa, J.; Valle-Tendero, F.; Cano, E.; Costa-Talens, M.; et al. Mapa de series, geoseries y geopermaseries de vegetación de España. Parte II. Itinera Geobot. 2011, 18, 425-800.

49. Salmerón-Sánchez, E.; Martínez-Nieto, M.I.; Martínez-Hernández, F.; Garrido-Becerra, J.A.; Mendoza-Fernández, A.J.; Gil de Carrasco, C.; Ramos-Miras, J.J.; Lozano, R.; Merlo, M.E.; Mota, J.F. Ecology, genetic diversity and phylogeography of the Iberian endemic plant Jurinea pinnata (Lag.) DC. (Compositae) on two special edaphic substrates: dolomite and gypsum. Plant Soil 2014, 374, 233-250. [CrossRef]

50. Medina-Cazorla, J.M.; Garrido-Becerra, J.A.; Mendoza Fernández, A.; Pérez-García, F.J.; Salmerón, E.; Gil, C.; Mota Poveda, J.F. 'Biogeography of the Baetic ranges (SE Spain): A historical approach using cluster and parsimony analyses of endemic dolomitophytes'. Plant Biosyst. 2010, 144, 111-120. [CrossRef]

51. Brković, D.L.; Tomović, G.M.; Niketić, M.S.; Lakušić, D.V. Diversity analysis of serpentine and non-serpentine flora-Or, is serpentinite inhabited by a smaller number of species compared to different rock types? Biologia 2015, 70, 61-74. [CrossRef]

52. Mota, J.F.; Garrido-Becerra, J.A.; Pérez-García, F.J.; Salmerón-Sánchez, E.; Sánchez-Gómez, P.; Merlo, E. Conceptual baseline for a global checklist of gypsophytes. Lazaroa 2016, 37, 7-30. 
53. Del Río, S.; Herrero, L.; Fraile, R.; Penas, A. Spatial distribution of recent rainfall trends in Spain (1961-2006). Int. J. Climatol. 2011, 31, 656-667.

54. Cano-Ortiz, A.; Musarella, C.M.; Piñar Fuentes, J.C.; Pinto Gomes, C.J.; Spampinato, G.; Cano, E. Taxonomy, ecology and distribution of Juniperus oxycedrus L. group in the Mediterranean Region using morphometric, phytochemical and bioclimatic approaches. BioRxiv 2018, 11, 1-23.

55. Santana, N.C. Fire Recurrence and Normalized Difference Vegetation Index (NDVI) Dynamics in Brazilian Savanna. Fire 2019, 2, 1. [CrossRef]

56. Spampinato, G.; Musarella, C.M.; Cano-ortiz, A.; Signorino, G. Habitat, occurrence and conservation status of the Saharo-Macaronesian and Southern-Mediterranean element Fagonia cretica L. (Zygophyllaceae) in Italy. J. Arid Land 2018, 10, 140-151. [CrossRef]

57. Liu, Y.; Zhao, W.; Zhang, X.; Fang, X. Soil water storage changes within deep profiles under introduced shrubs during the growing season: evidence from semiarid Loess Plateau, China. Water 2016, 8, 475. [CrossRef]

58. Liu, J.; Xiong, Y.; Tian, J.; Tan, Z. Spatiotemporal changes in evapotranspiration from an overexploited water resources basin in arid Northern China and their implications for ecosystem management. Sustainability 2019, 11, 445. [CrossRef]

59. Cano, E.; Musarella, C.M.; Cano-Ortiz, A.; Piñar, J.C.; Pinto Gomes, C.J.; Rodríguez Torres, A.; Spampinato, G. A phytosociological review of siliceous sedges in C-W Spain and their state of conservation based on diversity indices. Plant Sociol. 2017, 54, 5-14.

60. Piñar Fuentes, J.C.; Cano-Ortiz, A.; Musarella, C.M.; Pinto Gomes, C.J.; Spampinato, G.; Cano, E. Rupicolous habitats of interest for conservation in the central-southern Iberian Peninsula. Plant Sociol. 2017, 54, $29-42$.

61. Quinto-Canas, R.; Mendes, P.; Cano-Ortiz, A.; Musarella, C.M.; Pinto-Gomes, C. The Agrostion castellanae Rivas Goday 1957 corr. Rivas Goday \& Rivas-Martínez 1963 alliance in the southwestern Iberian Peninsula. Plant Sociol. 2018, 55, 21-29.

62. Spampinato, G.; Crisarà, R.; Cannavò, S.; Musarella, C.M. Phytotoponims of southern Calabria: A tool for the analysis of the landscape and its transformations. Atti Soc. Tosc. Sc. Nat. Mem. Serie B 2017, 124, 61-72.

63. Vila-Viçosa, C.; Vázquez, F.; Mendes, P.; del Rio, S.; Musarella, C.M.; Cano-Ortiz, A.; Meireles, C. Syntaxonomic update on the relict groves of Mirbeck's oak (Quercus canariensis Willd. and Q. marianica C. Vicioso) in southern Iberia. Plant Biosyst. 2015, 149, 512-526. [CrossRef]

64. Gratani, L.; Varone, L.; Bonito, A. Carbon sequestration of four urban parks in Rome. Urban For. Urban Green. 2016, 19, 184-193. [CrossRef]

65. Del Giudice, V.; Massimo, D.E.; De Paola, P.; Forte, F.; Musolino, M.; Malerba, A. Post Carbon City and Real Estate Market: Testing the Dataset of Reggio Calabria Market Using Spline Smoothing Semiparametric Method. In New Metropolitan Perspectives. ISHT 2018. Smart Innovation, Systems and Technologies; Calabrò, F., Della Spina, L., Bevilacqua, C., Eds.; Springer: Cham, Switzerland, 2019; pp. 206-214.

66. De Paola, P.; Del Giudice, V.; Massimo, D.E.; Forte, F.; Musolino, M.; Malerba, A. Isovalore Maps for the Spatial Analysis of Real Estate Market: A Case Study for a Central Urban Area of Reggio Calabria, Italy. In New Metropolitan Perspectives. ISHT 2018. Smart Innovation, Systems and Technologies; Calabrò, F., Della Spina, L., Bevilacqua, C., Eds.; Springer: Cham, Switzerland, 2019; pp. 402-410.

67. Malerba, A.; Massimo, D.E.; Musolino, M.; Nicoletti, F.; De Paola, P. Post Carbon City: Building Valuation and Energy Performance Simulation Programs. In New Metropolitan Perspectives. ISHT 2018. Smart Innovation, Systems and Technologies; Calabrò, F., Della Spina, L., Bevilacqua, C., Eds.; Springer: Cham, Switzerland, 2019; pp. 513-521.

68. Massimo, D.E.; Del Giudice, V.; De Paola, P.; Forte, F.; Musolino, M.; Malerba, A. Geographically Weighted Regression for the Post Carbon City and Real Estate Market Analysis: A Case Study. In New Metropolitan Perspectives. ISHT 2018. Smart Innovation, Systems and Technologies; Calabrò, F., Della Spina, L., Bevilacqua, C., Eds.; Springer: Cham, Switzerland, 2019; pp. 142-149.

69. Spampinato, G.; Massimo, D.E.; Musarella, C.M.; De Paola, P.; Malerba, A.; Musolino, M. Carbon sequestration by cork oak forests and raw material to built up post carbon city. In New Metropolitan Perspectives. ISHT 2018. Smart Innovation, Systems and Technologies; Calabrò, F., Della Spina, L., Bevilacqua, C., Eds.; Springer: Cham, Switzerland, 2019; pp. 663-671.

70. Vesterdal, L.; Schmidt, I.K.; Callesen, I.; Nilsson, L.O.; Gundersen, P. Carbon and nitrogen in forest floor and mineral soil under six common European tree species. Forest. Ecol. Manag. 2008, 255, 35-48. [CrossRef] 
71. Charro, E.; Moyano, A.; Cabezón, R. The potential of Juniperus thurifera to sequester carbon in semi-arid forest soil in spain. Forests 2017, 8, 330. [CrossRef]

72. Rădulescu, C.; Toader, R.; Boca, G.; Abrudan, M.; Anghel, C.; Toader, D.C. Sustainable Development in Maramures County. Sustainability 2015, 7, 7622-7643. [CrossRef]

73. Kurowska-Pysz, J.; Castanho, R.A.; Loures, L. Sustainable Planning of Cross-Border Cooperation: A Strategy for Alliances in Border Cities. Sustainability 2018, 10, 1416. [CrossRef]

74. Castanho, R.A.; Loures, L.; Cabezas, J.; Fernández-Pozo, L. Cross-Border Cooperation (CBC) in Southern Europe-An Iberian Case Study. The Eurocity Elvas-Badajoz. Sustainability 2017, 9, 360. [CrossRef]

75. Géhu, J.M.; Rivas-Martinez, S. Notions fondamentales de Phytosociologie. In Syntaxonomie; Dierschke, H., Ed.; Ber. Intern. Symposien IV-V; Cramer: Vaduz, Liechtenstein, 1981; pp. 5-33.

2019 by the authors. Licensee MDPI, Basel, Switzerland. This article is an open access article distributed under the terms and conditions of the Creative Commons Attribution (CC BY) license (http:/ / creativecommons.org/licenses/by/4.0/). 